\title{
Wireless on-demand drug delivery
}

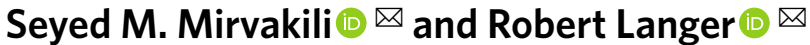

\begin{abstract}
Wireless on-demand drug delivery systems exploit exogenous stimuli-acoustic waves, electric fields, magnetic fields and electromagnetic radiation-to trigger drug carriers. The approach allows drugs to be delivered with controlled release profiles and minimal off-target effects. Recent advances in electronics and materials engineering have led to the development of sophisticated systems designed for specific applications. Here we review the development of wireless on-demand drug delivery systems. We examine the working mechanisms, applications, advantages and limitations of systems that are triggered by electric fields, magnetic fields or electromagnetic radiation. We also provide design guidelines for the development of such systems, including key metrics for evaluating the practicality of different smart drug delivery systems.
\end{abstract}

T he direct administration of drugs (by, for example, injection or orally) has traditionally been used for acute illnesses that require an immediate dose of a drug. For chronic diseases, where a sustained systemic or targeted release is required (such as for diabetes, psychogenic/psychiatric diseases and cancer), long-acting controlled release systems-including on-demand and smart drug delivery techniques-have been developed. Drugs can be controllably delivered by relying on the intrinsic physical or chemical properties of the target environment to trigger chemical carriers. However, on-demand drug delivery techniques, which have been explored over the past three decades, can offer better control over dosage, and the time and location of release.

Passive release techniques offer a predetermined release profile (sustained or extended) with poor control over the spatial distribution of the drug. These approaches are useful for specific conditions (such as disseminated diseases), but for localized diseases (such as tumours) active and targeted release mechanisms are needed to reduce the non-specific toxicity of the off-target sites and enhance the overall efficacy of the therapy. Smart drug delivery methods can provide the necessary spatiotemporal resolution and complex release profiles. To provide such capabilities, drug carriers have been engineered to release drugs in response to endogenous stimuli (such as $\mathrm{pH}$, concentration gradient, enzymes or the enhanced permeability and retention effect) and exogenous stimuli (such as electric field, magnetic field, electromagnetic field, temperature or ultrasound) at the target site.

On the basis of advances in electronics, actuators and materials engineering, powerful wireless on-demand drug delivery techniques have been created. These approaches exploit exogenous stimuli, including acoustic waves ${ }^{1-4}$, electric fields ${ }^{5,6}$, magnetic fields $s^{7,8}$ and electromagnetic radiation ${ }^{9-11}$, to trigger the drug carriers. Depending on the material and design of the drug carrier, the physiological characteristics of the target site, and the pharmacokinetics and pharmacodynamics of the cargo, each of these stimuli has particular applications. In this Review, we examine drug delivery systems (DDSs) that are operated with electric fields, magnetic fields and electromagnetic radiation. We restrict our analysis to approaches that do not require a physical medium for propagation. Acoustic excitation of DDSs is also an active research field, which uses mechanical waves, such as ultrasound, to trigger DDSs, and an analysis of such work can be found elsewhere ${ }^{1-4}$.

\section{Electric fields}

Electric fields can be generated by electrostatic charges, as in parallel plate capacitors, or time-varying magnetic fields, as in Joule heating in induction heating. They have been employed in medicine with different therapeutic effects such as actuating drug depots for in vivo drug delivery, enhancing the drug molecule transport via electroporation and iontophoresis, and even directly treating cancer tumours, called tumour-treating fields (TTFs) (Fig. 1a).

Actuating drug depots. Electric fields have been used to excite stimuli-responsive materials such as conducting polymers to release therapeutic payloads ${ }^{6}$. Conducting polymers are ionically and electronically conductive materials that can generate volumetric expansion/contraction upon electrochemical oxidation/reduction in an electrolyte ${ }^{12}$. Upon excitation with voltage, ion insertion or expulsion causes volumetric expansion or contraction of the polymer structure, respectively (Supplementary Information) ${ }^{12}$. The electrochemical reduction/oxidation enables the controlled release of the doped drug molecules (Supplementary Fig. 1a) ${ }^{5,13}$. Electroresponsive gels such as poly(dimethyl aminopropyl acrylamide) (PDMAPAA) loaded with drugs (for example, insulin) also have been demonstrated to release the cargo when stimulated by an externally applied electric field ${ }^{14}$. Similarly, hydrogels prepared from chitosan-graft-polyaniline copolymer and oxidized dextran loaded with amoxicillin/ibuprofen have shown a controllable release rate set by the applied voltage ${ }^{15}$. In these systems, the applied electric field also drives the charged molecules in the medium (electrophoresis), enhancing the overall efficacy ${ }^{14,15}$.

By harnessing the actuation properties of the conducting polymers, micro-/nanovalves have been fabricated to open and close nanopores of the drug reservoirs made of anodized aluminium oxide membrane (Fig. 1b) ${ }^{16}$. Unlike conducting polymers and hydrogels doped with drug molecules, this technique enables a sharp pulsatile release of drugs from large drug-depot systems ${ }^{16}$. Conducting polymers doped with drug molecules-in the form of nanodepot systems-have been shown to work with electric fields as well (Fig. 1c) ${ }^{13}$. These nano DDSs are injected subcutaneously and fixed in the tissue by mixing them with temperature-sensitive hydrogels before injection ${ }^{13}$. To enable the delivery of larger doses, macroscale depot systems in the form of a film can be administered in vivo and removed when depleted ${ }^{6,14}$.

Limitations. Application of electric fields to the depot systems is generally via one of two routes: sharp needle electrodes inserted into the dermis layer (invasive), where the depot is fixed in the tissue $^{13}$; applying metal pads on the surface of the skin (stratum corneum), above the depot ${ }^{14}$. The spatial arrangement of the 
Opening/closing pores
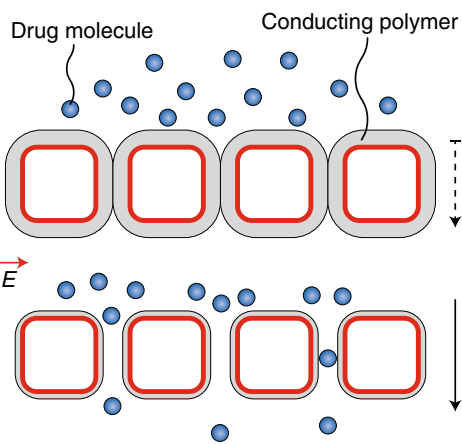

Deformation/actuation
Skin permeabilization
Electromigration and electroosmosis

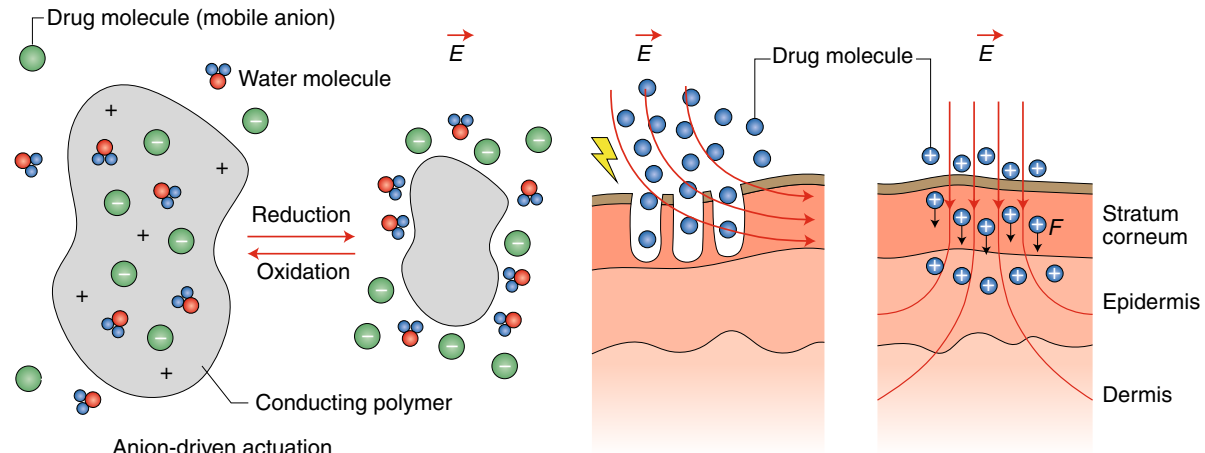

b

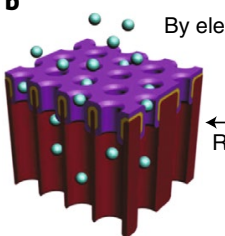

Open (oxidation state)

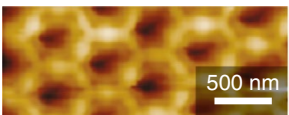

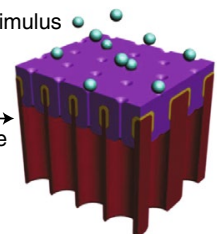

Close (reduction state)

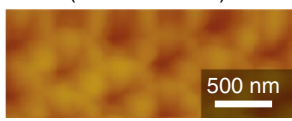
$\square \mathrm{Au}$ $c$ $\square$ PPy (DBS) $\square$ AAO O Drug

d $\overline{c_{3}}$ Biological anions
+ Biological cations O Neutral drug o Neutral analyte

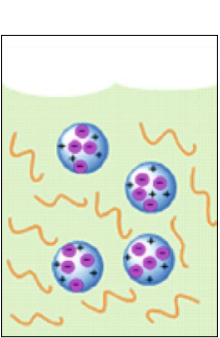

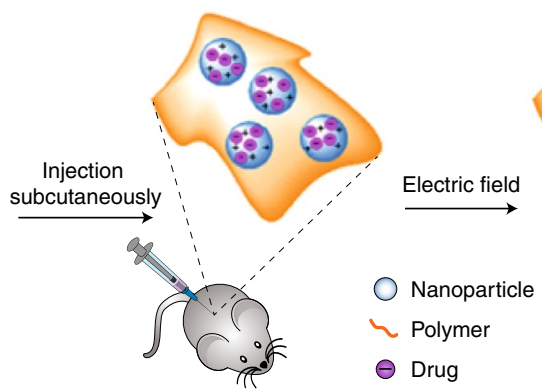

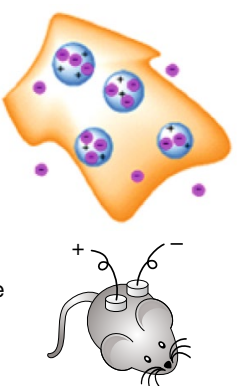

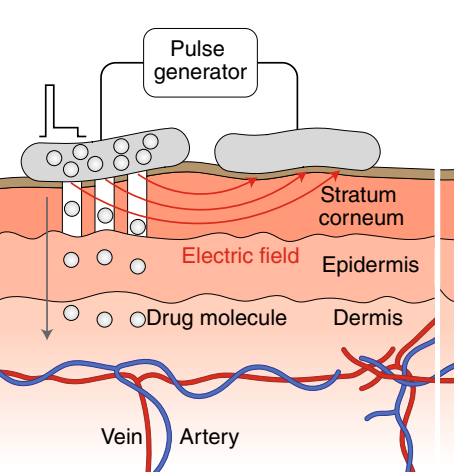

$$
\begin{aligned}
& \begin{array}{c}
\text { Microcontroller } \\
\text { and } \\
\text { current sensor }
\end{array} \\
& \hline
\end{aligned}
$$

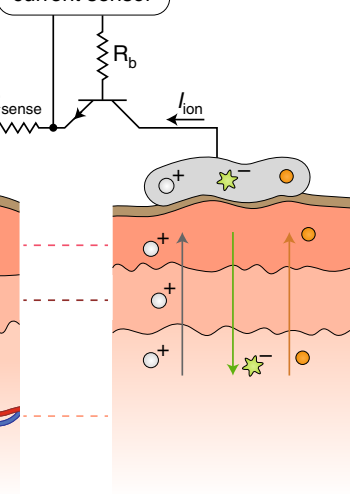

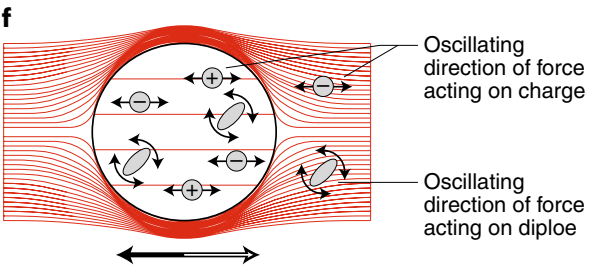

Field of alternating direction

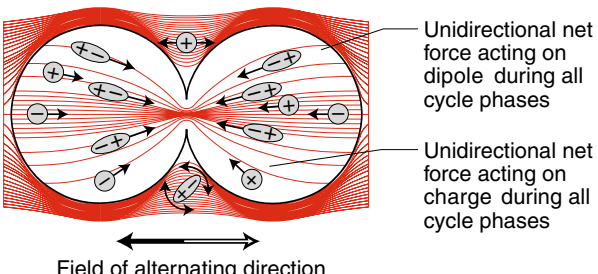

Fig. 1 | DDSs and electric fields. a, Four mechanisms for exciting DDSs with electric fields ( $E$ ). Left: electric fields open pores of a drug reservoir covered with conducting polymers. Middle left: conducting polymer nanoparticles prepared with negatively charged drug molecules. When excited with electric fields, the nanoparticles undergo reduction reactions that shrink the nanoparticles and release the drug molecules (other forms described in the Supplementary Information). Middle right: application of the electric field increases the skin permeability via electroporation. Right: electric field enhances the transportation of surface-charged small-molecule drugs by exerting force $(F)$ on the ions. b. Electrically actuatable smart nanoporous membrane made of polypyrrole (PPy) doped with dodecylbenzenesulfonate (DBS) for pulsatile drug release. Top: reversible change of pore size (and the drug release rate) between oxidation and reduction states. Bottom: in situ atomic force microscopy height images corresponding to the oxidation and reduction states. $A A O$, anodized aluminium oxide. c, Electric-field-responsive nanoparticles loaded with a drug. From left to right: the nanoparticle-polymer solution is subcutaneously injected into a mouse, followed by application of a d.c. electric field to induce the release of the drug cargo inside the nanoparticles. d, Electroporation working principle. Dielectric breakdown at the stratum corneum perforates the skin surface, increasing the permeability of the skin. Right after, the voltage is lowered to circumvent stimulation of the underlying tissues. e, lontophoresis working principle. A low-current signal is applied at the skin's surface $\left(I_{\text {ion }}\right)$ to transport the ions across the skin via electrophoresis/electromigration of surface-charged small-molecule drugs and then electroosmotic flow. $V+$ is the applied voltage, $R_{\text {sense }}$ is the sense resistor and $R_{b}$ is the resistor connected to the base of the transistor. $\mathbf{f}$, TTFs working principle. Alternating current field distribution (red lines) in and around quiescent (top) and dividing (bottom) cells ${ }^{43}$. The forces exerted by the electric field on the ions (in grey) are shown with arrows. Figure reproduced with permission from: b, ref. ${ }^{16}$, American Chemical Society; c, ref. ${ }^{13}$, American Chemical Society; f, ref. ${ }^{43}$, National Academy of Sciences. 
electrodes/DDSs as well as the local concentration of electrolytes and ionizable molecules notably affect the responsiveness of the DDSs ${ }^{17}$. One of the challenges associated with using electric fields in biological systems is the electric double layer formation at the electrodes' surface. In electrochemically conductive electrolytes, the distance at which the potential decays to $1 / \mathrm{e}$ of its value at the electrode is called the Debye length $\left(\kappa^{-1}\right)$. The typical value for the Debye length is on the order of a few nanometres for simple electrolytes (for example, low-concentration saltwater). In other words, in only a few nanometres from the electrodes, the potential decays to $36 \%$ of its value at the electrodes (Supplementary Fig. 1b).

Due to the high nonlinearity in the potential profile, it is difficult to measure and control the electric field inside the tissue. Therefore, it would be more accurate to measure and control the current, as the ratio between the applied voltage and the distance between the electrodes can overestimate the electric field. Unless a reference electrode is used, constant current (galvanostatic) excitation of such DDSs is a more reliable way of achieving reproducible and accurate results for a two-electrode system.

Electroporation and iontophoresis. In topical and transdermal drug delivery, electric fields can enhance the efficacy via electroporation and iontophoresis. In electroporation, high-voltage pulses (typically $>100 \mathrm{~V}$ ) with pulse width on the order of microseconds to milliseconds are applied to the surface of the skin to permeabilize it (Fig. 1d). The process has been shown to reversibly disrupt the cell membranes (for gene transfection) and the lipid bilayer structures in the $\operatorname{skin}^{18,19}$. During the excitation period, electrophoretic migration is the dominant transport mechanism. In the off-cycle, diffusion through long-lived electropores is the primary transport mechanism (for hours). This combination increases the transdermal transport by orders of magnitude for DNA, vaccines, peptides and small-molecule drugs ${ }^{20,21}$. In fact, this technique has shown some promising initial results for delivering the coronavirus disease

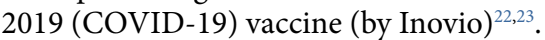

The electrical resistance of the skin (stratum corneum layer) is orders of magnitude higher than that of the deeper tissues. Thus, during excitation, there is a large potential drop across the stratum corneum until electroporation occurs. Right after, the resistance drops rapidly by a large factor, which increases the potential drop across the deeper tissues (for example, epidermis, dermis, hypodermis). This potential gradient often results in the stimulation of the muscular tissue, nerves and sweat glands. Closely spaced microelectrodes have been shown to resolve the issue by decreasing the electric-field path length and constraining it within the stratum corneum $^{24}$. Controlling the current with a fast response control system/ mechanism can prevent painful stimulation of the deeper tissues. Similar control schemes are used in the ballasts of arc lamps to limit the current rapidly to compensate for the rapid drop in the resistance (from the megaohm range to the few ohm range) of the lamp after the arc formation ${ }^{25}$.

A typical topology for generating low-current and kilovoltrange-voltage pulses is high-voltage d.c.-to-d.c. converters. One of the low-cost (<US\$450) and straightforward designs for such d.c.-to-d.c. converters is based on an EMCO1 module and is controlled via a microcontroller (for example, Arduino) ${ }^{26}$. This design has been successfully demonstrated for exciting dielectric elastomer actuators at $5 \mathrm{kV}, 1 \mathrm{mHz}$ to $1 \mathrm{kHz}$ (ref. ${ }^{26}$ ).

In iontophoresis, low-voltage (typically $<10 \mathrm{~V}$ ) galvanostatic (constant current) excitation of electrodes $\left(0.5 \mathrm{~mA} \mathrm{~cm}^{-2}\right)$ at the surface of the skin transports the drug across the stratum corneum layer (Fig. 1e $)^{27}$. Unlike electroporation, the iontophoresis process barely alters the skin's permeability; thus, the main driving force is electrophoresis/electromigration of surface-charged small-molecule drugs and then electroosmotic flow ${ }^{28}$. Molecules without sufficient electric charges are transported via an electroosmotic flow of water generated by mobile ions in the stratum corneum ${ }^{29}$. Iontophoresis typically works only for small molecules. However, transdermal delivery of macromolecules up to a few kilodaltons has been shown with this technique ${ }^{20}$.

Due to the nature of the transport mechanism in iontophoresis, the flux of drug (often in $\mu \mathrm{g} \mathrm{cm}^{-2} \mathrm{~h}$ ) in the stratum corneum layer scales with the electric charge transfers at the surface of the electrodes (often in $\left.\mathrm{mA} \mathrm{cm}^{-2}\right)^{30}$. Therefore, by integrating the amount of charge circulated in the circuit, the total delivered dose can be determined. For precision delivery, this task is achieved either by using a microcontroller (with a Coulomb counter), powered by a battery ${ }^{31}$ / energy harvesting systems $\mathrm{s}^{32}$, or using a battery with a known charge capacity (with some simple controllers) ${ }^{33}$.

Examples of iontophoresis for transdermal drug delivery include rapid delivery of lidocaine for local anaesthesia ${ }^{34}$, tap water for hyperhidrosis treatment ${ }^{35}$, pilocarpine for cystic fibrosis diagnosis ${ }^{36}$, fentanyl for pain relief ${ }^{37}$, acyclovir for herpes labialis treatment ${ }^{31}$ and extraction of glucose for glucose monitoring ${ }^{38}$. Aside from transdermal drug delivery, ophthalmic drug delivery with iontophoresis has shown to be a promising approach for drug delivery. For example, transscleral iontophoresis of dexamethasone phosphate has demonstrated dramatically enhanced intraocular concentrations of dexamethasone in ocular tissues of rabbits compared with topical instillation ${ }^{39}$.

In a different application scheme, iontophoresis has shown potential implications for treating pancreatic, breast and other solid tumours $^{40,41}$. For example, it has been demonstrated that in vivo iontophoresis of gemcitabine to pancreatic cancer tumours leads to a mean $\log _{2}$ fold change in tumour volume of -0.8 (ref. ${ }^{40}$ ).

Advantages. Iontophoresis provides notable control over the dosage of delivery due to its straightforward working mechanism. Therefore, it can be used to generate complex release profiles on demand without requiring complicated feedback circuits.

Limitations. Due to the slowness of the transport across the stratum corneum (that is, minutes to hours), the small window of currents/ voltages (microampere to milliampere range) for safe operation and shallow depth of delivery, iontophoresis is most effective if it is combined with other methods that can increase skin permeability (for example, electroporation, ultrasound $)^{41,42}$. This combination possibly improves transdermal delivery of macromolecules or vaccines ${ }^{42}$.

Tumour-treating fields. TTFs (by Novocure) is an emerging non-invasive treatment modality that uses alternating electric fields (average $1 \mathrm{~V}_{\text {r.m.s. }} \mathrm{cm}^{-1}$ and 100 to $300 \mathrm{kHz}$ ) (Supplementary Fig. 1c) to reduce the tumour growth rate by interfering with the cell division process (mitosis) ${ }^{43}$. At present, this technique does not involve delivering therapeutic cargos but rather achieves its therapeutic effects by using a small amplitude of intermediate-frequency electric fields alone. Regardless, we are briefly introducing this powerful technique to pose possible integration of this technology with the wireless drug delivery techniques that we subsequently discuss in this Review.

In TTF therapy, the force generated by the electric field on the cell structure proteins interferes with the chromosome separation process, thereby inducing cell cycle arrest and apoptosis (cell suicide) of only rapidly dividing cancer cells (quiescent and non-dividing cells are unaffected) (Fig. If and Supplementary Fig. 1d ${ }^{43,44}$. TTFs have been demonstrated to provide powerful clinical benefit in glioblastoma multiforme, one of the three most common types of brain tumour ${ }^{44}$. When combined with TTF therapy, an increase in sensitivity to chemotherapy has been shown in pre-clinical studies ${ }^{45}$. In addition to glioblastoma multiforme, TTFs have been shown to suppress the proliferation of cells of different cancer types at different optimal frequencies (Supplementary Fig. 1e). 
Limitations. While TTF therapy is proven to be highly effective ${ }^{43,44}$, like many other treatments, it comes with mild to moderate adverse effects such as scalp irritation and headache below the transducer array and adhesive bandage ${ }^{44}$. TTF therapy is an active field of research, and more on the efficacy of this technique for treating different tumours is a topic that yet needs to be investigated.

\section{Magnetic fields}

One of the advantages of the magnetic field over the electric field for application in biological systems is that it can deeply penetrate the tissue with minimal interaction with the ions (non-invasive). These important features have enabled applications in imaging (magnetic resonance imaging), diagnosis and targeted drug delivery. Utilization of magnetic fields, from static and low-frequency fields $(<20 \mathrm{kHz})$ to high-frequency fields $(>100 \mathrm{kHz})$, in DDSs have shown to be a promising technique for micro- and nanomedicine.

Static and low-frequency magnetic fields. Static and low-frequency magnetic fields can be generated by magnets or electromagnets. A basic electromagnet consists of a coil with a magnetic core and a d.c. power supply. The magnetic-field strength scales with the current passing through the coil linearly. Static magnetic fields have been used to mechanically deform soft scaffolds, infiltrated with ferromagnetic materials, to expel therapeutic cargo embedded within their structure ${ }^{46,47}$. This technique has been shown to effectively release therapeutic payloads on demand in vitro and in vivo (Fig. 2a $)^{46-48}$. Nevertheless, any mechanical deformation induced from inside or outside the body can accidentally release the content. To address this issue, liposomes (loaded with chemotherapeutic regimens) have been engineered to release drug on demand when exposed to a low-frequency $(10 \mathrm{kHz})$ alternating magnetic field ${ }^{49}$. This engineered structure consists of chains of magnetic nanoparticles attached to the liposomes. Under a $10 \mathrm{kHz}$ magnetic field, the vibration from the chains disrupts the membranes and releases the drug to the tumour (Fig. 2b) ${ }^{49}$.

Similar to electrophoresis and iontophoresis, magnetic fields have shown the potential to enhance transdermal drug delivery. In magnetophoresis, a magnet $(<450 \mathrm{mT})$ is placed at the skin's surface to repel diamagnetic hydrophilic drug molecules across the stratum corneum or even across cell membranes in deeper tissues ${ }^{50}$. For example, it has been demonstrated that the permeability of vascular endothelium can be increased when infiltrated with iron oxide nanoparticles and stimulated with an external static magnetic field ${ }^{51}$. The force on the magnetic nanoparticles (MNPs) temporarily disrupts the cell membranes, thus enhancing the transportation of the substances ${ }^{51}$. Unlike iontophoresis, in magnetophoresis, the drug molecules' net charge does not affect the transportation rate. Similarly, ions and charged particles in the tissue are not perturbed during magnetic excitation, making it a safe solution for enhancing transdermal drug delivery of hydrophilic drugs ${ }^{50}$.

Aside from drug delivery, low-frequency magnetic fields have been used to navigate micro-/nanorobots (also known as magnetic swimmers) for drug delivery and minimally invasive therapeutic and diagnostic medical procedures (Fig. 2c) ${ }^{52-54}$. These magneto-responsive microcarriers can reproduce the motions of natural swimming microorganisms with active flagella ${ }^{55}$. The nanorobots have been shown to deliver therapeutic payloads effectively (for example, plasmid DNA) ${ }^{56}$ directly to the cells across the cellular membranes. Aside from synthetic magneto-responsive carriers, it has been shown that magneto-aerotactic bacteria, Magnetococcus marinus strain MC-1-swimmers that travel naturally in the direction of Earth's magnetic-field lines-can be magnetically guided to deliver therapeutic cargos. They have been shown to transport drug-loaded nanoliposomes into hypoxic regions of tumours to enhance the penetration depth and the overall efficacy ${ }^{57,58}$. Magnetic guiding of micro-/nanorobots is an emerging field with a wide range of potential applications. Various novel designs have been explored for delivering therapeutic cargos, as described in the recent review works on this topic ${ }^{54,55,59}$; however, there is more room for integration with other drug delivery techniques.

Advantages. The mechanism of generating static and low-frequency magnetic fields is straightforward. Moreover, compared with electric and electromagnetic fields, magnetic fields have insignificant interactions with biological tissues making it safe for various applications, including navigating magnetic-based DDSs to the site of action.

Limitations. Bulky, heavy and often expensive coils or magnets are needed to generate the required magnetic-field strengths for triggering the DDSs inside the body. This size requirement for generating the triggering stimulus limits their portability. Thus, patients must visit medical centres to activate the DDS(s), which renders the on-demand aspect usable in only certain facilities.

High-frequency magnetic fields. Alternating magnetic fields can be generated by applying an alternating current through a coil or a rotating magnet. At high frequency $(>20 \mathrm{kHz})$, magnetic-field oscillation can generate heat in metals and magnetic particles. The heat generation occurs via different mechanisms depending on the materials' magnetic/electrical properties and size. Metals with a grain size larger than $1 \mu \mathrm{m}$ generate heat due to eddy current formation in response to alternating magnetic fields ${ }^{60}$. The eddy current generates a Joule heating effect, which scales with the square of the frequency (that is, $f^{2}$ ), the square of the magnetic-field strength (that is, $H^{2}$ ) and the grain diameter mean square (that is, $\left.\left\langle d^{2}\right\rangle\right)^{60}$. For single-domain MNPs (for example, superparamagnetic nanoparticles-often less than $30 \mathrm{~nm}$ in size), Brownian (physical alignment of the nanoparticles) and Néel (alignment of the magnetic moment within the nanoparticles) fluctuations are responsible for heat generation (Supplementary Fig. 2a) ${ }^{60}$. For multidomain MNPs, hysteresis losses $\left(\propto H^{3}\right.$ and $\left.\propto f\right)$ are the dominant heating mechanism ${ }^{60}$.

Multiple approaches have been employed to utilize highfrequency magnetic fields in drug delivery and hyperthermia for tumour ablation ${ }^{7,8,61,62}$. One of the most promising designs uses MNPs to release therapeutic cargos from micro/nano hollow capsules (for example, liposomes) ${ }^{63-65}$ or solid particles (for example, polymer based) ${ }^{66-68}$. For example, MNPs (such as $\mathrm{Fe}_{3} \mathrm{O}_{4}, \gamma-\mathrm{Fe}_{2} \mathrm{O}_{3}$ ) embedded in drug-loaded biodegradable polymers, such as poly(lactic-co-glycolic acid) (PLGA), have been shown to trigger and release the cargo when excited with alternating magnetic fields $(100 \mathrm{kHz} \text { to } 200 \mathrm{kHz})^{66}$. When heated, the polymer network undergoes structural rearrangements, which increases the diffusion rate of the drug (Fig. 2d). With this technique, pulsatile release profiles have been demonstrated ${ }^{66}$.

Another design has been proposed that employs thermal triggering of lower critical solution temperature (LCST) hydrogels (for example, poly $(N$-isopropylacrylamide) (pNIPAM)), made of networks of crosslinked long polymer chains ${ }^{69}$, to facilitate drug release. LCST hydrogels have a combination of hydrophobic and hydrophilic segments in their polymer chains. At temperatures below the LCST, the hydrogels swell due to the domination of hydrophilic interactions with water and absorb the drug ${ }^{12}$. However, at temperatures above the LCST, the hydrogen bonds with water are broken, and hydrophobic interactions between the polymer chains dominate ${ }^{12}$, resulting in the deswelling of the hydrogel and the release of the cargo (Fig. 2e) $)^{70}$. This technique has been demonstrated for on-demand 'burst' release of drugs and bioactive proteins for stem-cell proliferation ${ }^{71,72}$.

A third design employs the magneto-thermal effect to open the 'gates' or pores of drug reservoirs. The gates can be made of thermosensitive hydrogels ${ }^{73,74}$ or just by producing defects in the 


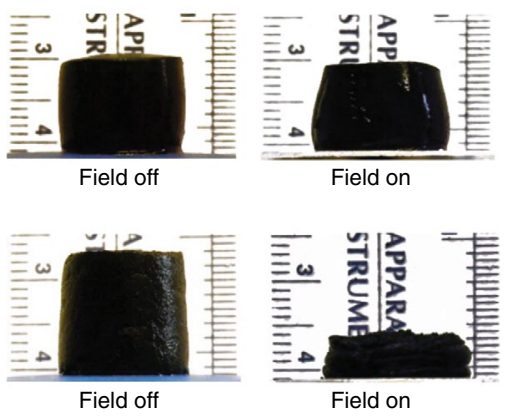

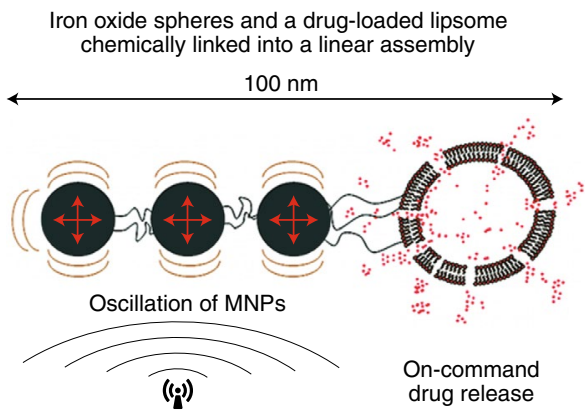

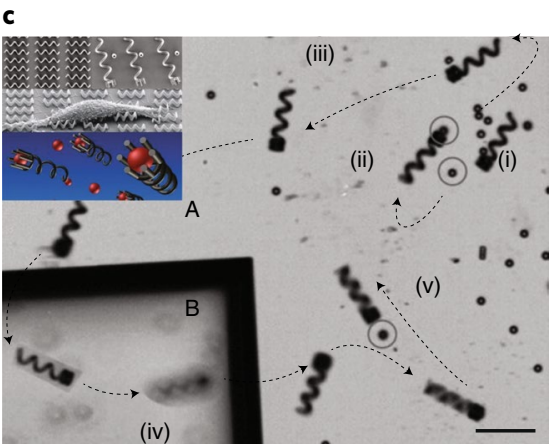

d

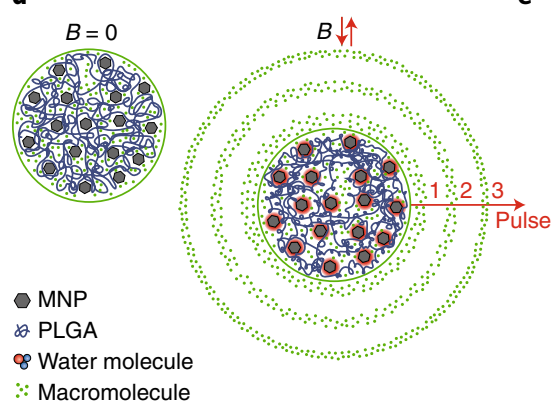

e

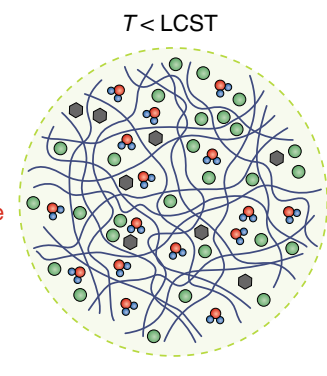

$\mathbf{f}$

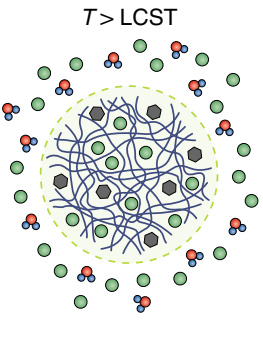

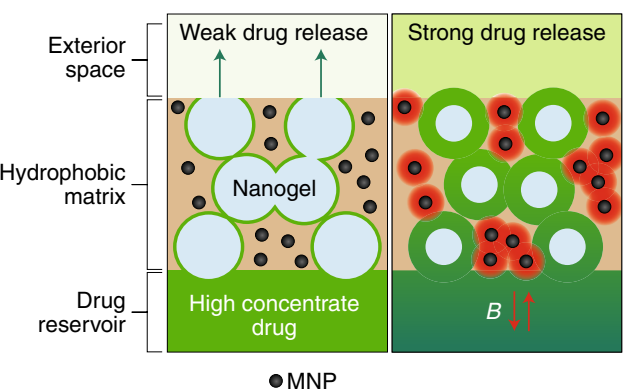

g

○ MNP
$\because$ Drug

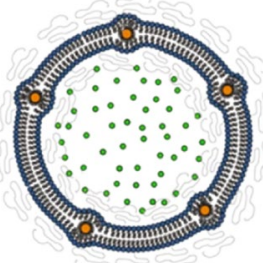

h

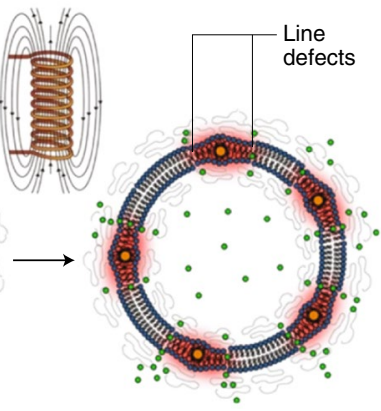

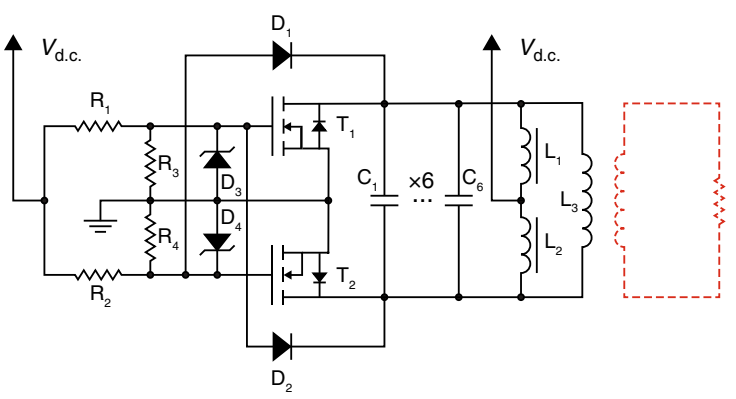

i

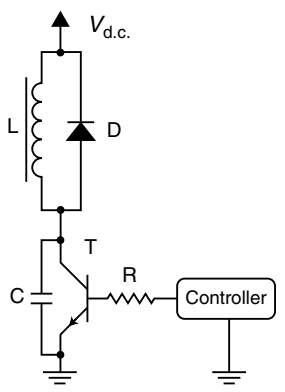

Fig. 2 I DDSs and magnetic fields. a, Static magnetic-field excitation of a DDS. Top: a cylinder of a nanoporous ferrogel reduced its height by $\sim 5 \%$ when subjected to a vertical magnetic-field gradient of $\sim 38 \mathrm{~A} \mathrm{~m}^{-2}$. Bottom: the corresponding macroporous ferrogel deformed by $\sim 70 \%$ under the same magnetic field ${ }^{47}$. b. In vitro evaluation of the RF-triggered release profile of doxorubicin from doxorubicin-nanocarrier particles. Illustration of the defects on the liposome caused by 'vibration' (red arrows) of the iron oxide spheres under an RF field. c, Time-lapse image of the pick-and-place micromanipulation of a 6- $\mu \mathrm{m}$-diameter microparticle. The circles indicate the selected microparticle. The trajectory is shown with labels (i) to (v). The stepped surface has two levels, and A indicates the higher level and B the lower one. Scale bar, $50 \mu \mathrm{m}$. d, Illustration of the release mechanism. When excited with alternating magnetic field $(B)$, the temperature of the polymer increases, which leads to an increase in the mobility of the polymer chains. Therefore, the free volume inside the network increases, enabling a higher diffusion rate of the macromolecules. e, For temperatures ( $T$ ) below the LCST, the hydrogel swells, while above the LCST, it shrinks and releases the cargo. f, Proposed schematic of a cross-section of the membrane, showing nanogel particles (blue), iron oxide nanoparticles (black) and ethylcellulose matrix (light brown). Upon application of a magnetic field, the magnetic nanoparticles release heat (red) and reversibly shrink the nanogel, enabling release of a drug (green) from a reservoir contained by the membrane. $\mathbf{g}$, Local heat generation from superparamagnetic iron oxide nanoparticles through Néel's relaxation produces a line defect in the membrane separating melted (liquid) from solid domains $s^{64} \cdot \mathbf{h}$, Circuit schematic of a high-frequency $(100 \mathrm{kHz})$ alternating magnetic-field generator made of resistors $\left(R_{1}-R_{4}\right)$, inductors $\left(L_{1}-L_{3}\right)$, transistors $\left(T_{1}, T_{2}\right)$, diodes $\left(D_{1}-D_{4}\right)$ and capacitors $\left(C_{1}-C_{6}\right)$. $V_{\text {d.c. }}$, voltage. Magnetic nanoparticles can be modelled as the secondary winding of a 'transformer' with the primary winding being the induction coil. $\mathbf{i}$, Circuit schematic of an electromagnet. Figure reproduced with permission from: $\mathbf{a}$, ref. ${ }^{47}$, National Academy of Sciences; b, ref. ${ }^{49}$, American Chemical Society; c, ref. ${ }^{134}$, Wiley; d, ref. ${ }^{66}$, American Chemical Society; $\mathbf{f}$, ref. ${ }^{74}$, American Chemical Society; $\mathbf{g}$, ref. ${ }^{64}$, under a Creative Commons licence CC BY 4.0; $\mathbf{h}$, ref. ${ }^{60}$, AAAS.

membrane by locally melting/degrading it (Fig. 2f,g) ${ }^{64}$. This technique generates a burst release, and unlike the other two designs, the drug molecules are minimally heated. Moreover, drugs over a wide range of molecular weight $(500-40,000 \mathrm{Da})$ can be delivered ${ }^{74}$.

Generating alternating magnetic fields involves fast switching of a coil of an LC (inductor-capacitor) resonator. A typical topology used for generating high-frequency $(<500 \mathrm{kHz})$ magnetic fields is based on zero-voltage switching. To reduce the switching noise and the voltage/current stress on the metal-oxide-semiconductor field-effect transistor (MOSFET) during on-off transitions, soft switching is used using a MOSFET with a fast body diode across its drain and source (Fig. 2h,i). For higher frequencies $(>500 \mathrm{kHz})$, combining a signal generator with a radiofrequency (RF) power amplifier is a better solution. 
Advantages. The drug carriers with MNPs can be spatially guided via static magnetic fields to increase the delivery efficacy. When at the target site, the release can be either via a passive diffusion process $^{75}$ or high-frequency magnetic-field excitation of the carrier ${ }^{70}$. The latter can be combined with hyperthermia to increase the therapeutic efficacy for certain cases ${ }^{61}$.

A magnetic field, at low intensities $\left(H \times f<4.85 \times 10^{8} \mathrm{~A} \mathrm{~m}^{-1} \mathrm{~s}\right.$ $\left.{ }^{-1}\right)^{61,76}$, is a non-invasive stimulus and can be generated and controlled easily by low-cost electronics (Supplementary Information). The magnetic-field intensity scales with the inverse of the coil diameter ${ }^{60,66}$. As stated previously, the effective heating power per mass of MNPs scales with $f$ or $f^{2}$ (depending on the materials and its size). Therefore, by miniaturizing the coil (for example, planar microcoils) ${ }^{77}$ (Supplementary Fig. 2b) and exciting the coils at higher frequencies, a similar heating effect can be expected ${ }^{66}$. Such miniaturization can potentially enable small devices that can trigger micro/nano DDSs inside the body for complications with irregular and unpredictable occurrence of symptoms (for example, pain, blood coagulation, psychotic disorders) ${ }^{66}$.

In addition to drug delivery and hyperthermia, high-frequency magnetic fields have been employed for the non-invasive treatment of prosthetic joint infections ${ }^{78}$ and also nuclear magnetic resonance imaging ${ }^{79,80}$. Magnetically controlled DDSs is an emerging field in nanomedicine that unveils a landscape of opportunities for imaging, targeted in vivo drug delivery and therapy in a multimodal fashion $^{46,66,74,81}$.

Limitations. At the moment, there is no direct way of measuring the exact temperature of the MNPs inside the body. Thus, a very precise and well-trained open-loop control system is needed to control the magnetic-field intensity/frequency to circumvent any excessive heating of the drug and the surrounding tissue and the induction of off-target effects. By engineering the Curie temperature of the MNPs, it is possible to set a maximum temperature limit for induction heating of the MNPs and minimize necrosis and hypothermic shock to healthy tissues.

It is important to note that only specific drug molecules can tolerate the heat generated by the MNPs. In fact, heat can have deleterious effects on proteins and some vaccines. Finally, the therapeutic payloads can leach or leak from the carrier due to passive diffusion and cause complications.

\section{Electromagnetic radiation}

Synchronized oscillations of electric and magnetic fields generate electromagnetic waves (or their quanta, photons). Electromagnetic radiation covers a wide span of wavelengths, including radio waves and microwaves, infrared, visible light, ultraviolet, $\mathrm{X}$-rays and gamma-rays (Fig. 3). These frequency bands offer unique properties for DDSs and other medical devices, some of which have not been explored yet.

Radio waves and microwaves. RF waves are generated by the acceleration of charged particles in a conductor (for example, an alternating current in an antenna). The spectrum for radio waves covers as low as $30 \mathrm{~Hz}$ to $300 \mathrm{GHz}$ with corresponding wavelengths of $10,000 \mathrm{~km}$ to $1 \mathrm{~mm}$, respectively. The $1 \mathrm{GHz}$ to $100 \mathrm{GHz}$ spectrum is often defined as microwaves. There are two frequency bands under the Medical Implant Communication Service (MICS) approved by the Federal Communications Commission for medical implants and body-worn medical devices: the $401-457 \mathrm{MHz}$ band, with a total bandwidth of $29 \mathrm{MHz}$, and the $2,360-2,400 \mathrm{MHz}$ band, specifically for a medical body area network (MBAN) (Fig. 3) ${ }^{82}$. RF waves have been utilized in DDSs in two general approaches. The first involves using RF waves for communication and powering DDSs, and the second uses RF waves for exciting DDSs (Fig. 4a).

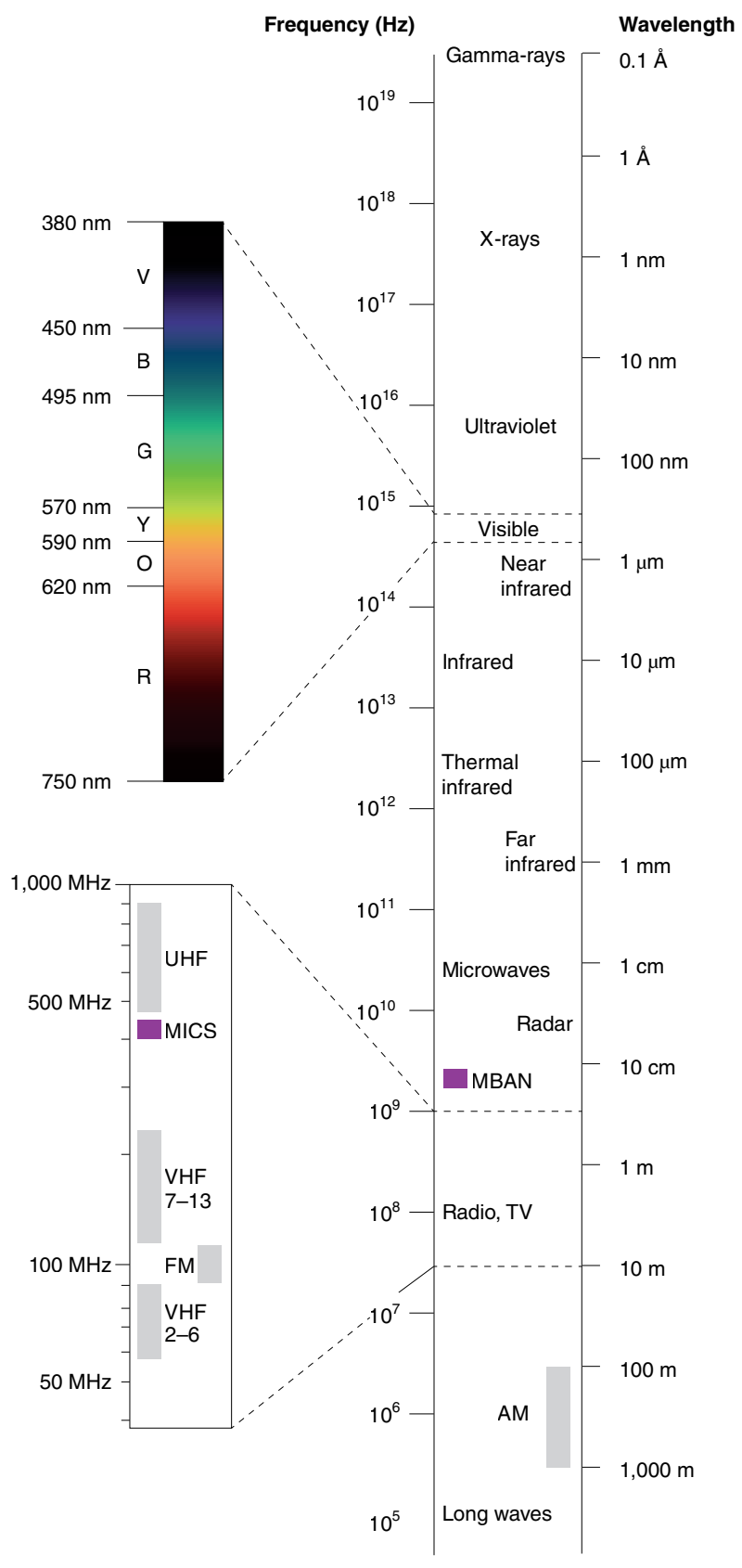

Fig. 3 | Electromagnetic radiation spectrum. In radio communication, amplitude modulation (AM) is typically used for wavelengths from $100 \mathrm{~m}$ to $1 \mathrm{~km}$. Frequency modulation (FM) is typically used around $100 \mathrm{MHz}$ frequency. The ultra-high frequency (UHF) range can span from $300 \mathrm{MHz}$ to $3 \mathrm{GHz}$. The Medical Implant Communication System (MICS) is designated for communication with medical implants. Very high frequency (VHF) ranges from $30 \mathrm{MHz}$ to about $300 \mathrm{MHz}$ and has three major bands (VHF 2-6, FM and VHF 7-13). The visible range includes red (R), orange $(O)$, yellow $(Y)$, green $(G)$, blue $(B)$ and violet $(V)$.

$R F$ waves for communication and powering DDSs. By utilizing similar microfabrication processes in making solid-state electronics, microchips have been fabricated with on-chip drug reservoirs, a wireless transmitter and a microcontroller to generate on-demand complex drug release profiles (Fig. $4 \mathrm{~b}-\mathrm{e})^{83,84}$. This technique precludes micropumps and valves and works on a simple dispensing mechanism (electrothermal ${ }^{85}$ or electrochemical ${ }^{86}$ dissolution of the reservoirs' membranes). Moreover, it offers a very precise 


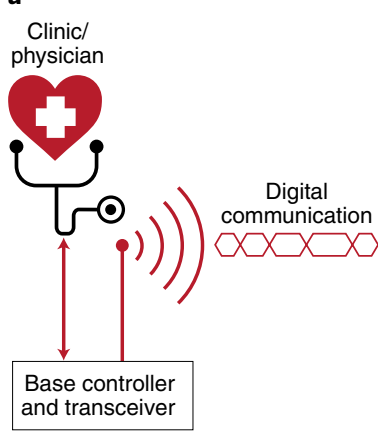

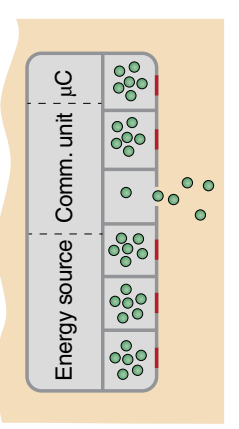
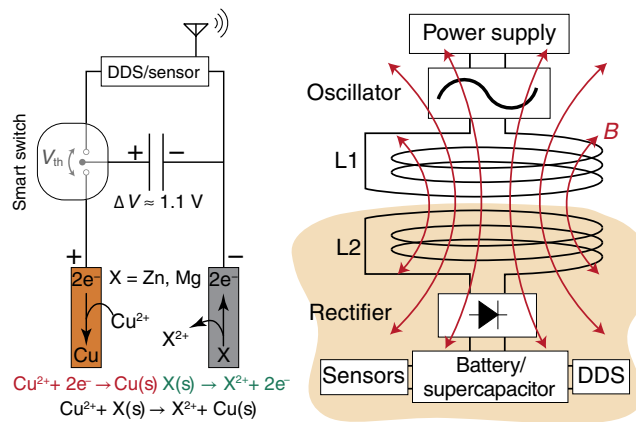

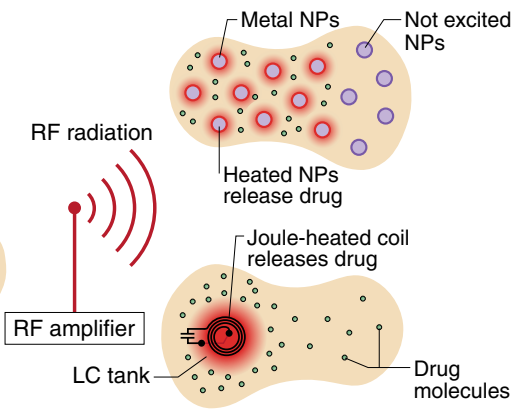

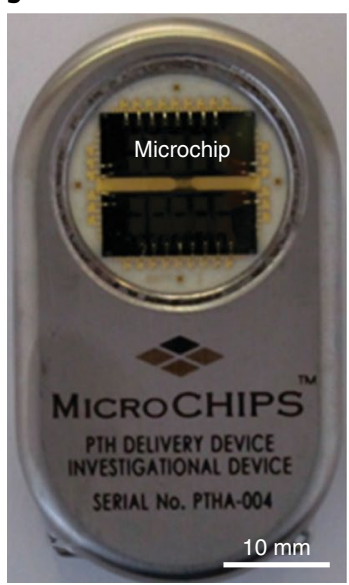
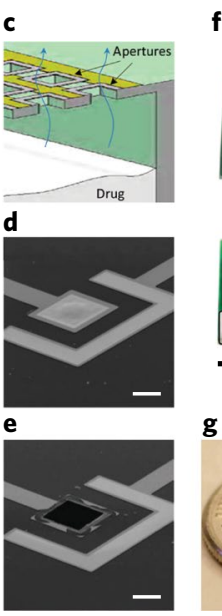

f

8

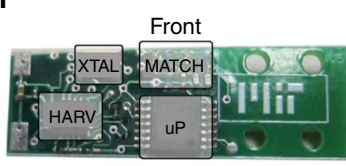

Reverse
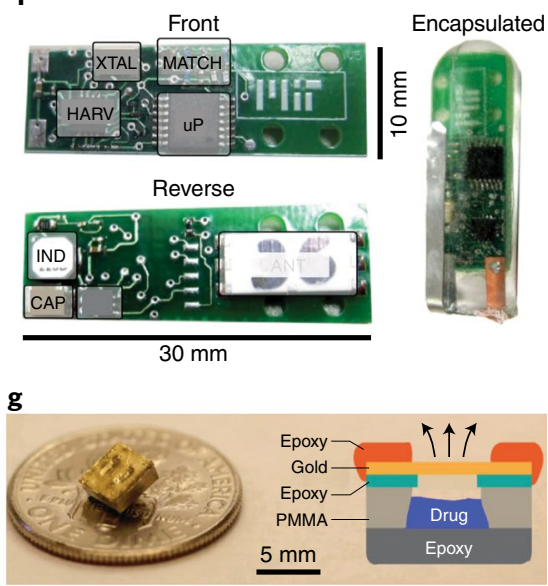

h

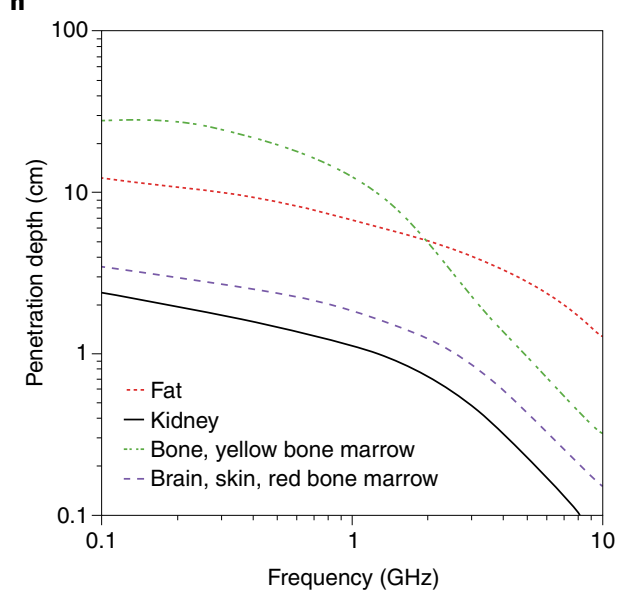

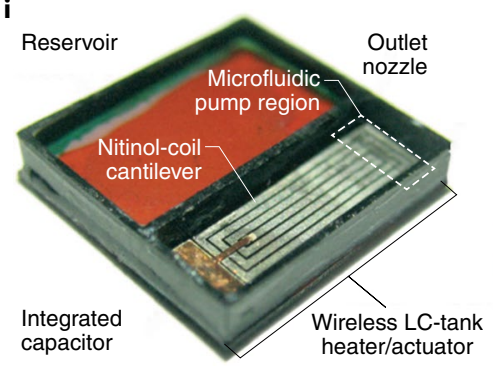

j
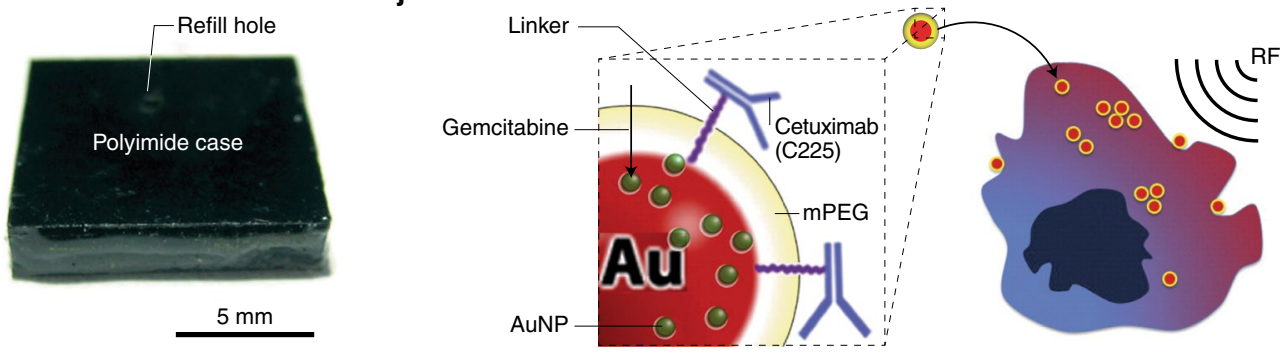

Fig. 4 | DDSs and electromagnetic fields (radio waves). a, Left: wirelessly controlled DDS with an on-board microcontroller ( $\mu$ C). Middle left: energy harvesting from a galvanic cell. When a threshold voltage $\left(V_{t h}\right)$ is reached, the capacitor releases the energy to the DDS circuit. Middle right: wireless power transfer via magnetic coupling between two coils (L1, L2). Right: wireless excitation of drug carriers. b, Microchip-based human parathyroid hormone fragment (1-34) (hPTH(1-34)) drug delivery device ( $54 \mathrm{~mm} \times 31 \mathrm{~mm} \times 11 \mathrm{~mm}$, length $\times$ width $\times$ height). c, Schematic cross-section of microchip assembly showing drug release from one reservoir. $\mathbf{d}, \mathbf{e}$, Removal of an anode membrane to initiate release from a reservoir. Scanning electron micrographs of a gold membrane anode covering a reservoir are shown before (d) and after (e) the application of $+1.04 \mathrm{~V}$ with respect to the saturated calomel electrode (SCE) for several seconds in phosphate-buffered saline. Scale bars, $50 \mu \mathrm{m}$. $\mathbf{f , g}$, Demonstration of galvanic-cell-powered gastric temperature measurement, wireless transmission and DDS. $\mathbf{f}$, Images of the circuit board with each module highlighted. $\mu \mathrm{P}$ is the microprocessor, MATCH is the antenna matching circuit, XTAL is the crystal oscillator, HARV is the energy harvesting unit, CAP is a capacitor and IND is an inductor. The encapsulated circuit board is shown on the right. $\mathbf{g}$, The drug reservoir structure and its relative size to one dime is shown. Polymethyl methacrylate (PMMA) is used to make the walls of the reservoir. $\mathbf{h}$, Penetration depth of RF fields in body tissues depending on frequency and tissue type (logarithmic scales). $\mathbf{i}$, The nitinol LC wireless resonant heater is integrated into the chip to perform cantilever actuation for drug delivery. Fabricated drug delivery chip without (left) and with (right) polyimide casing. j, Gemcitabine-loaded, epidermal growth factor receptor (EGFR)-targeted AuNPs for drug delivery and non-invasive RF field-induced hyperthermia. The particle shell is made of methoxypoly(ethylene glycol) (mPEG). Figure reproduced with permission from: b,c, ref. ${ }^{84}$, AAAS; d,e, ref. ${ }^{83}$, Springer Nature Ltd; f,g, ref. ${ }^{94}$, Springer Nature Ltd; i, ref. ${ }^{101}$, RSC; $\mathbf{j}$, ref. ${ }^{106}$, Elsevier. Panel h adapted with permission from ref. ${ }^{108}$, EMF-Portal.

spatiotemporal release profile. With this method, it has been shown that human parathyroid hormone can be delivered daily, tested for 20 days, inside the body with better release-profile consistency than injections ${ }^{84}$. This DDS implant has a power consumption of $<10 \mathrm{~mW}$ and runs on a battery (Supplementary Fig. 3a-c) ${ }^{85}$. It wirelessly communicates (amplitude-shift keying (ASK) modulation in the $402-405 \mathrm{MHz}$ MICS band) with the base station, controlled by a physician, to report the device's status and receive the dosing/ release schedule ${ }^{84}$. The silicon-based pharmacy-on-a-chip is not biodegradable and requires removal at the end of its cycle life. This issue has been addressed by fabricating the chip from bioresorbable materials and biological lipid membranes ${ }^{87-89}$.

Two main approaches have been proposed to eliminate the need for batteries for DDS implants: wireless power transfer and on-board 
energy harvesting. One of the standard techniques in non-radiative wireless power transfer involves transferring energy via two induction coils through near-field inductive coupling. The device is simply a transformer with its primary and secondary windings separated by a short distance (Fig. 4a). This wireless power transfer mechanism has been shown to enable complete wireless operation of the pharmacy-on-a-chip device discussed previously ${ }^{90,91}$. However, it requires precision impedance matching between the coils and is extremely sensitive to the relative movement and distance between the two coils. These shortcomings have been addressed to some extent recently ${ }^{92,93}$ and should be implemented in wireless implants to be tested for real-life scenarios.

On-board energy harvesting has been shown to be a promising technique for DDSs residing inside the body for prolonged periods. Examples include harvesting the galvanic corrosion energy of metal (zinc, magnesium, aluminium)-copper galvanic cells (Fig. 4f,g) ) $^{94-96}$ or harvesting high-frequency $(>800 \mathrm{MHz})$ electromagnetic waves (Supplementary Fig. 3d,e). The first category utilizes a rechargeable battery in combination with a capacitor to sense/release drugs and transmits signals wirelessly to the outside of the body ${ }^{94}$. The latter is still new and progress yet needs to be made for complete human-body implementation and clinical trials. In short, it has been previously shown that Wi-Fi signals can be harvested to power up sensors ${ }^{97}$. The attenuation of high-frequency signals in tissue and the limits set by the Federal Communications Commission for RF transmission power through tissue has made it difficult for implementation in DDSs for in vivo applications. A new approach has been proposed to resolve this issue by utilizing multiple antennas (phased arrays) to form and steer beams electronically using constructive and destructive interference between sources ${ }^{98}$.

$R F$ waves for exciting DDSs. Alternative approaches to the pharmacyon-a-chip technology have been proposed in which an electromagnetic wave is used to excite micropumps (for example, infusion, peristaltic, osmotic, displacement $)^{99}$ and microvalves ${ }^{100}$ on demand to facilitate drug delivery from drug reservoirs. For example, it has been demonstrated that by making an LC resonator coil out of $\mathrm{NiTi}$ (shape memory alloy) microwires and thermally exciting it with RF magnetic fields, the drug can be pumped out of the reservoir on demand (Fig. $4 \mathrm{i})^{101}$. Thermal actuators are often inefficient and low bandwidth ${ }^{12}$. Moreover, thermal excitation can have adverse effects on tissues if not appropriately managed. To address these issues, wirelessly powered microvalves made of piezoelectric actuators have been proposed to release drugs on demand rapidly (similar to the ink-dispensing mechanism in inkjet printers) ${ }^{102}$.

The relatively large size (millimetre range) of the pharmacyon-a-chip technology and its invasive implanting process can be costly and uncomfortable for patients. To overcome these issues, it has been demonstrated that it is possible to open the membranes/ lids by attaching ring oscillators and exciting them with $2.45 \mathrm{GHz}$ RF signals ${ }^{103}$.

At smaller scales, to enhance the penetration of the DDSs to tissues and cells, nanoparticles (for example, gold, carbon nanotubes, iron-based MNPs, platinum and quantum dots) have been employed $^{104-106}$. Aside from carrying therapeutic cargos (for example, antibodies) to the site of action, cancer cell ablation via heating has been demonstrated with these particles. For example, it has been shown in a model of hepatocellular carcinoma (one of the most lethal and chemo-refractory cancers) that non-invasive RF-induced hyperthermia combined with cetuximab targeted delivery of a gold nanoparticle (AuNP)-gemcitabine conjugate is more safe and effective at dosages $\sim 275$-fold lower than the current clinically used systemic dose of gemcitabine (Fig. $4 \mathrm{j})^{106}$.

The heating mechanism below the optical frequency range is different from the surface plasmon resonance occurring in the optical frequency range and is not well understood yet ${ }^{107}$. One of the

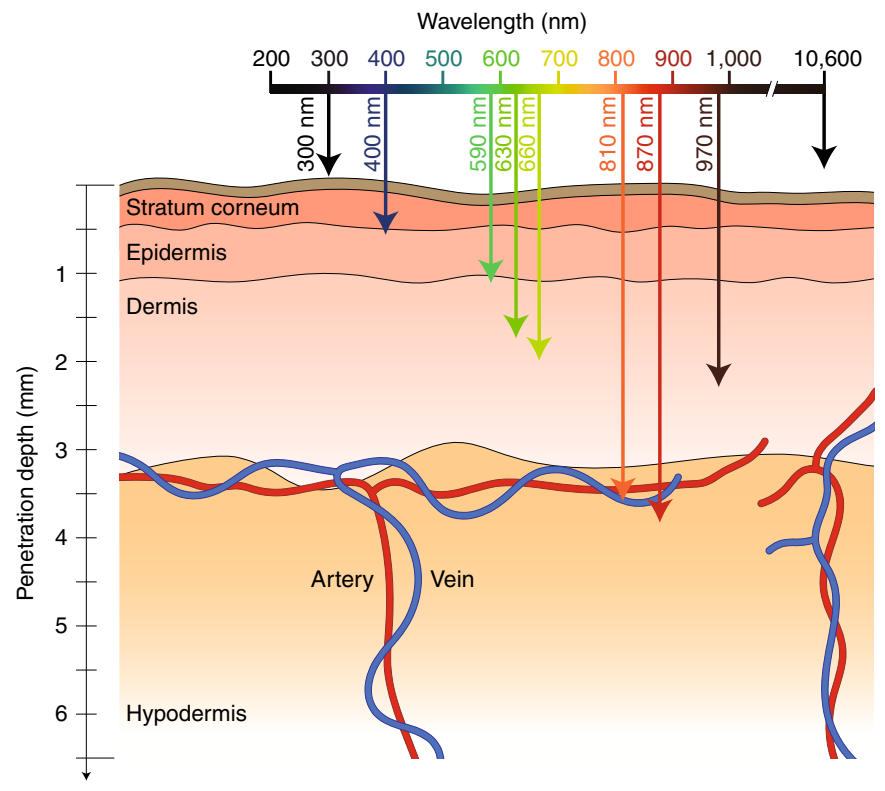

Fig. 5 | Schematic illustration of light penetration depth as a function of frequency in skin tissue. Figure adapted with permission from ref. ${ }^{107}$, American Chemical Society.

advantages of operating below the optical frequency range is the better penetration depth the microwave range offers compared with the near infrared (NIR) ${ }^{107}$.

Advantages. RF operated systems are easy to implement, low cost and offer robust controllability over the release profile. Moreover, RF circuits can be very small and operate at low power, making them an excellent candidate for more commercially viable DDSs and other devices for medical care.

Limitations. Radio waves and microwaves interact with dipole molecules (for example, water)/charge carriers and induce rotation, vibration and/or translation in the media (for example, body fluid, tissues containing water and salts). These nanoscale activities generate friction between molecules and/or charge carriers of other atoms, thus heating up the media (Supplementary Information). To minimize heating of healthy tissues, the amplitude and frequency of the radio waves/microwaves should be carefully chosen. As a result of these electromagnetic wave-matter interactions, the penetration depth of electromagnetic waves is less in organs with higher water or salt contents (for example, bones versus liver) (Fig. 4h) ${ }^{108}$. Therefore, techniques, such as phased arrays, should be developed to increase the wireless energy transfer with no adverse effects on the healthy tissues.

The shape and size of the antenna dictate its optimal operating frequency. For a simple antenna (for example, long wire), the length should be equal to the half-wavelength $(\lambda / 2)$ of the electromagnetic wave (Supplementary Information). The higher the frequency, the shorter/smaller the antenna can be designed.

One of the major challenges with the implant DDSs (for example, microchip) is the requirement for the replenishment of the payloads ${ }^{83,84}$. Currently, the whole system needs to be removed from the body, filled with a new drug cartridge and placed back in the target $\operatorname{site}^{83,84}$. It would be useful to develop implant DDSs that can receive the drug non-invasively or to develop approaches to refill them (for example, as in an Ommaya reservoir).

Infrared. The infrared spectrum is from $700 \mathrm{~nm}(430 \mathrm{THz})$ to $1 \mathrm{~mm}$ $(300 \mathrm{GHz})$. Light penetration in skin tissue is maximum $(\sim 4-5 \mathrm{~mm})$ 
around $870 \mathrm{~nm}$ (NIR) (Fig. 5) ${ }^{109}$. This property has been used to make infrared-sensitive DDSs. Heat generation due to surface plasmon resonance (Fig. 6a,b) in metal nanoparticles (for example, gold ${ }^{110}$, iron oxide ${ }^{111}$, ruthenium ${ }^{112}$ ) has been exploited to trigger drug release from thermosensitive polymers. The design of such DDSs is very similar to those discussed in the section 'High-frequency magnetic fields' with the exception that the energy-converting units are different (AuNPs versus MNPs) and are excited with electromagnetic radiation as opposed to alternating magnetic fields ${ }^{113-116}$.

Aside from directly exciting the DDSs, infrared radiation can generate side reactions that can trigger the release mechanism. For example, photosynthesizers can generate reactive oxygen species when exposed to NIR radiation to facilitate the release of drugs from DDSs (Fig. 4a,c) ${ }^{117-119}$. Like magnetophoresis and electrophoresis, infrared radiation has been shown to enhance transdermal drug delivery via direct ablation, photomechanical waves and the photothermal effect ${ }^{120}$.

Advantages. Light has a non-invasive nature and is easy to generate and use. More importantly, it offers high spatial resolution and temporal control, which enable sequential triggering of multiple payloads with high accuracy.

Limitations. For deep tissue, light-in the infrared, visible or ultraviolet spectrum-is not an ideal trigger for DDSs. The photon conversion efficiency of the optical receivers on DDSs still needs to be enhanced to enable activation at low light intensities ${ }^{121,122}$. High-power lasers and light sources can have detrimental effects on the skin and the underlying layers ${ }^{121}$. The biocompatibility and biodegradability of the photoresponsive elements in the DDSs are still not fully addressed for in vivo applications ${ }^{10}$. A very limited number of photoresponsive components have been explored so far, which are not scalable for the real-world clinical and translational applications yet. Considering the open-loop nature of the technique, it is desirable to have some form of feedback such as imaging agents, to help identify the site of action (for example, tumour) and prevent off-target damage.

Visible light. Skin penetration is less in the visible light spectrum than in the infrared spectrum but has been shown to be effective enough to trigger DDSs. By tuning the diameter of AuNPs to their plasmonic resonance wavelength (for example, $532 \mathrm{~nm})^{123}$, it has been shown that surface plasmon resonance can trigger the release of drug molecules from the nanoparticles (Fig. 6d) ${ }^{123,124}$. Alternative approaches involve drug release based on the decomposition of light-responsive organic moieties (for example, vitamin B12 derivatives, trithiocarbonates) or transition metal compounds (for example, ruthenium complexes) trapping the drug molecules when exposed to light in the visible spectrum ${ }^{125,126}$. A similar trapping mechanism has been explored with poly(ethylene glycol)-polylactic acid (PEG-PLA) for intravenous treatment of choroidal neovascularization with phototargeted nanoparticles ${ }^{127}$.

Advantages. Visible light offers similar advantages to the infrared spectrum.

Limitations. The visible spectrum has a smaller penetration depth compared with the infrared spectrum, but still there is a limited number of platforms that can utilize visible light as a trigger for DDSs.

Ultraviolet. Ultraviolet radiation carries enough energy $(\sim 4 \mathrm{eV}$ to $300 \mathrm{eV}$ ) to alter molecular bonds leading to a reconfiguration of the molecular structure. This energy level can be harmful to the human body as it can lead to mutation and cause cancer. However, on the low-energy side of the spectrum and at low intensities, it has been shown to be useful in triggering drug release from DDSs.

There are a handful of polymers that reversibly shrink when exposed to ultraviolet light. For example, azobenzenes undergo cis-trans photoisomerization when exposed to ultraviolet light and contract $^{12,128}$. Similarly, photoreversible [2+2] cycloaddition reactions in polymers containing cinnamic groups have been shown to cause the polymers to contract under ultraviolet irradiation ${ }^{129}$. Spiropyran-based nanoparticles have been demonstrated to contract by about $52 \%$ (103 nm to $49 \mathrm{~nm}$ ) when excited with $365 \mathrm{~nm}$ ultraviolet light (photoisomerization between spiropyran and merocyanine $)^{130}$. This property has been implemented in nano DDSs to enhance the release and tissue penetration of the drug (Fig. 6e $)^{130}$.

The penetration depth of ultraviolet in the skin is less than $1 \mathrm{~mm}$, which reduces the excitation rate of DDSs in deep tissues. Wireless micro/nano ultraviolet sources can enable local excitation of DDSs at the site of action by minimizing damage to the healthy tissues.

Advantages. Ultraviolet light offers similar advantages to the visible and infrared spectrum plus it is energetic enough to break chemical bonds (for example, between a drug molecule and a carrier/ nanoparticle).

Limitations. The high-energy property of ultraviolet also sets a limit on its application as it has detrimental effects on cells at the DNA level and can only be used in brief bursts. Moreover, it has a very small penetration depth $(<1 \mathrm{~mm})$. All in all, selecting the right spectrum for the phototriggered DDSs is the choice between the degree of manipulation needed versus the penetration depth in the tissue.

Fig. 6 | DDSs and electromagnetic fields (infrared to gamma). a, Left: NIR-triggered $\mathrm{O}_{2}$ releasing and enhanced photodynamic therapy mechanism. Middle left: light-triggered surface plasmon heating of AuNPs releasing the attached drug molecules. Middle right: light-triggered molecular reconfiguration of the drug carriers enables the release of the payload. Right: light-induced cavitation. For example, light-induced singlet oxygen generation cavitates the liposomal membrane and releases the drug. b, Graphical illustration of plasmons in bulk gold (left) and NPs (right). Left: bulk plasmons can be excited by an electron beam (bottom), while propagating surface plasmon polaritons (PSPPs) are excited by the evanescent field of light (top). Right: localized surface plasmons (LSPs) are excited by light propagating in free space or dielectric media (top). The LSP in AuNPs can be modelled, as a first approximation, like a spring-mass harmonic oscillator, where the free-electron density is the equivalent of the mass (bottom). c, Schematic illustration of NIR-triggered $\mathrm{O}_{2}$ releasing and enhanced photodynamic therapy (PDT) mechanism in a metal-organic framework nanoplatform for highly efficient PDT against a hypoxic tumour. d, Visible-light-triggerable aptamer/hairpin DNA-AuNP (apt/hp-AuNP) conjugate for targeted drug delivery. Aptamer $\mathrm{Sgc} 8 \mathrm{c}$ is a short DNA sequence that can target protein tyrosine kinase 7 (PTK7). e, Scheme of the photoswitching of spiropyran and its nanoparticulate formulation. Top: structure and photoisomerization reaction between spiropyran (SP) and merocyanine (MC). Bottom: scheme of photoswitchable SP/ DSPE-PEG lipid hybrid nanoparticles (NPHs). DSPE, 1,2-distearoyl-sn-glycero-3-phosphorylethanolamine. f, Schematic illustration of gene silencing and cancer-cell-killing by X-ray-triggered liposomes. This liposomal delivery platform incorporates verteporfin (VP) and AuNPs. Two types of cargo, antisense oligonucleotide and doxorubicin, are respectively entrapped inside a liposomal middle cavity for demonstration of in vitro gene release and in vivo drug delivery. Figure reproduced with permission from: b, ref. ${ }^{135}$, IOP; c, ref. ${ }^{118}$, Elsevier; d, ref. ${ }^{123}$, American Chemical Society; e, ref. ${ }^{130}$, National Academy of Sciences; f, ref. ${ }^{132}$, under a Creative Commons licence CC BY 4.0 . 
$\mathrm{X}$-ray. X-ray is most often used in radiation therapy for treating cancer (for example, skin, brain, lung, prostate, breast) ${ }^{131}$. Owing to its high radiation energy, $124 \mathrm{eV}$ to $124 \mathrm{keV}$ ( $10 \mathrm{pm}$ to $10 \mathrm{~nm}$-atomic scale), and considerable penetration depth, X-ray has been widely used for imaging of inner organs in the body. X-rays are generated via the interaction of highly accelerated electrons with electrons of tungsten atoms within a vacuum tube. Aside from radiotherapy and imaging, recently, it has been proposed to use X-rays to trigger drug release in tumours to achieve synergistic effects combined with the standard radiotherapy ${ }^{132}$. The technique involves incorporating AuNPs and verteporfin photosensitizer into drugloaded liposomes ${ }^{132}$. Under $6 \mathrm{MeV} \mathrm{X}$-ray radiation, verteporfin generates singlet oxygen, disrupting the liposomal membrane and releasing the cargos (Fig. 6f) ) $^{132}$.

Advantages and limitations. Owing to the X-ray radiation's considerable penetration depth, this technique offers prospects for wireless triggering of nanodrug carriers in deep tissues. However, the negative side-effects of X-ray radiation on healthy tissue must be considered to maximize its benefits.

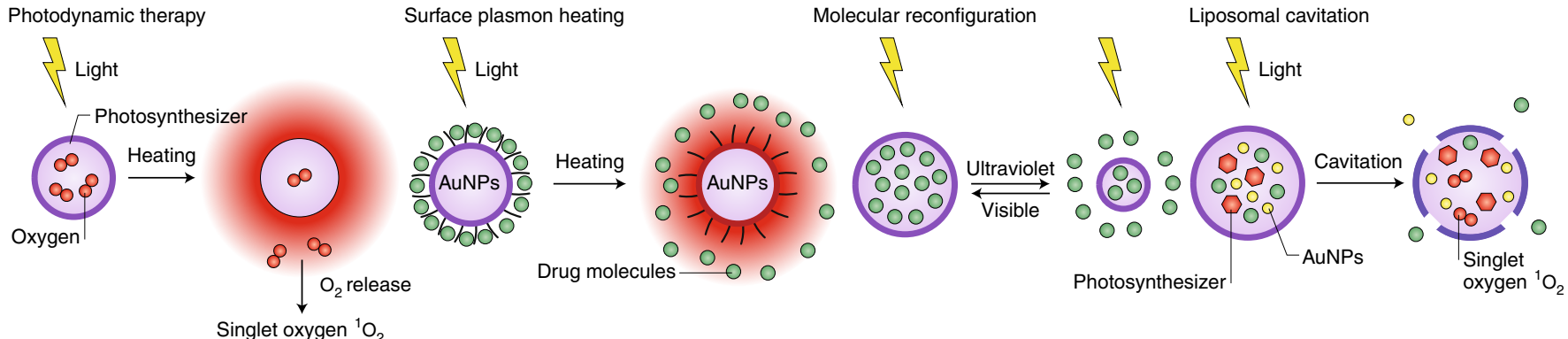

b
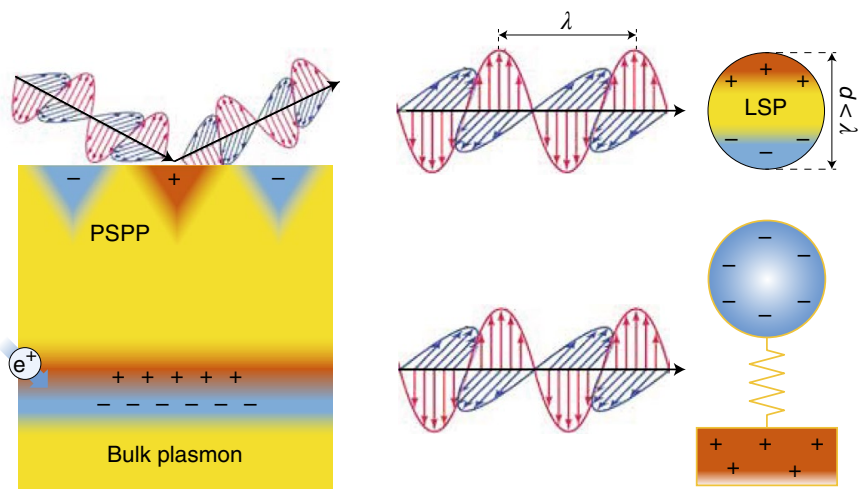

d

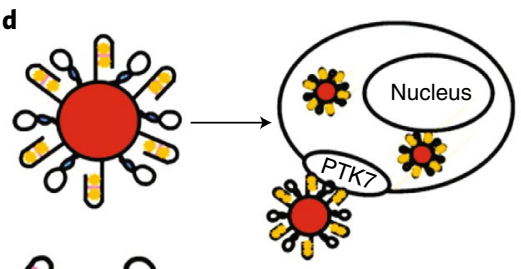

$\gamma_{\text {hpDNA }}$ q Sgc8c $_{\text {Doxorubicin }}$

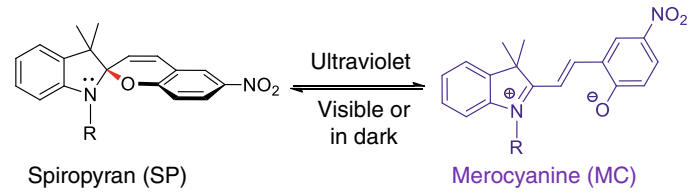

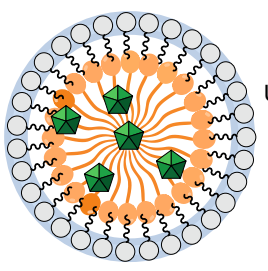

SP NPHs

\section{Ultraviolet $(365 \mathrm{~nm})$}

\section{Visible or}

in dark

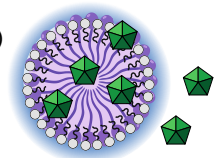

MC NPHs
Q
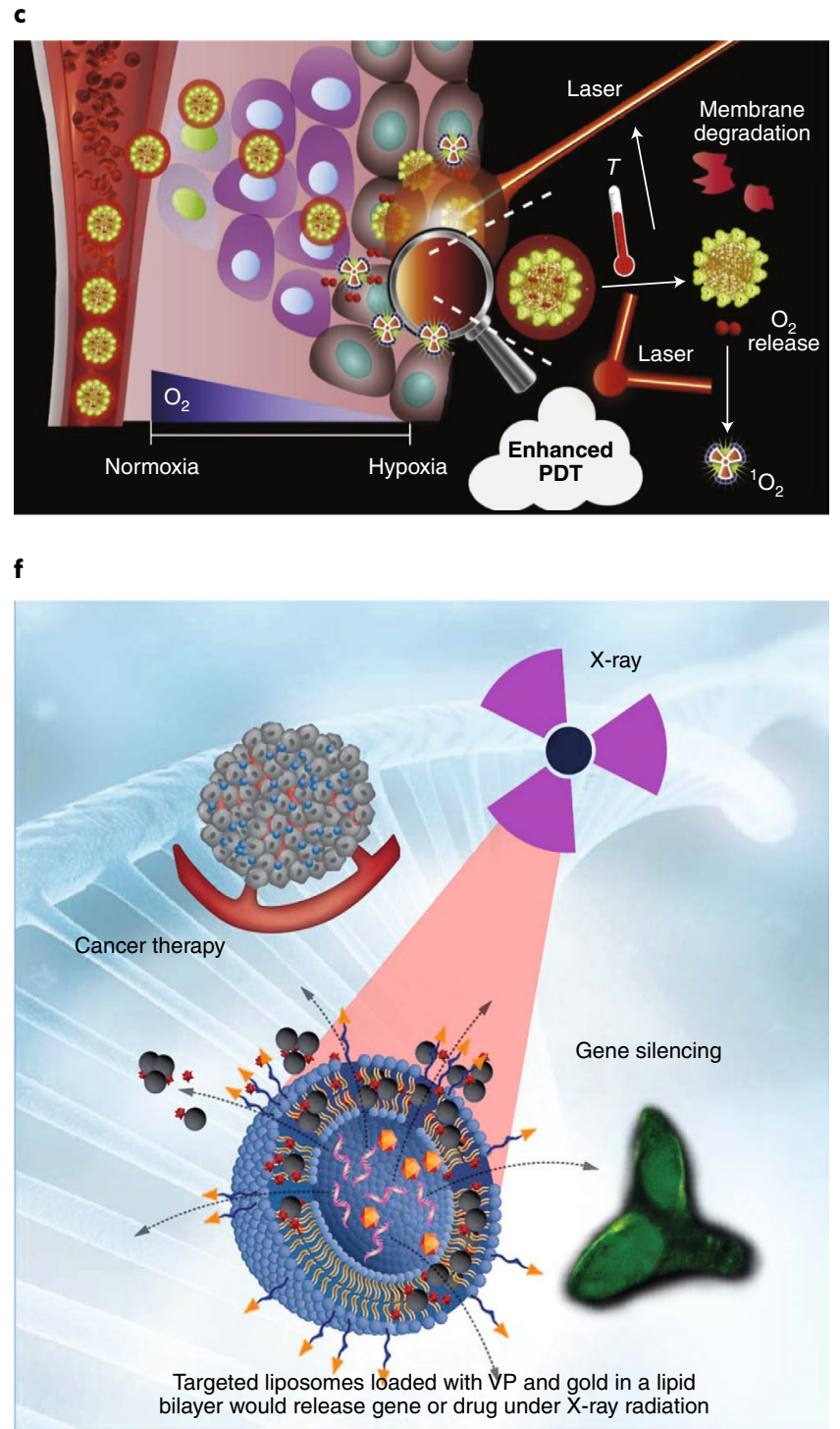
Gamma-rays. Gamma-rays are emitted by the change in the energy state of the nucleus of atoms, which is distinct from X-rays, where the transition in energy states of electrons is responsible for radiation. Gamma-rays are generated through radioactive decay of nickel-56 and cobalt-56 and have energy levels from a few kiloelectronvolts to $8 \mathrm{MeV}$ (wavelengths of atomic nuclei scale). Due to the extremely high energy levels of gamma-ray radiation and its adverse effects on biological tissues, at present, no DDS has been made for use with gamma-rays. However, recently it has been shown that gamma-rays can be used to sterilize nano DDSs (for example, chitosan microparticles, liposomes, niosomes, sphingosomes), with almost no side-effects, before administration ${ }^{133}$.

Compared with electric and magnetic fields, electromagnetic radiation can be collimated easily (for example, lasers, with reflectors) to increase the radiation intensity without increasing the input power to the source. However, electromagnetic radiation is absorbed by the skin at specific wavelengths, generating adverse effects on healthy tissues.

\section{Design guidelines}

Depending on the application of DDSs, different metrics are used to evaluate their suitability. These metrics are more meaningful for DDSs that are tested in vivo. While there are many elegant designs in the literature reporting high-efficacy DDSs tested in vitro, their performance must be evaluated in vivo to obtain a realistic opinion about their pros and cons. The most important metrics that are commonly used to evaluate the practicality of a DDS include the following.

Biocompatibility. The safety and biocompatibility of some of the elements used to make remotely triggerable DDSs (for example, photosynthesizers, thermoresponsive hydrogels, photoswitchable organic molecules) need to be studied further for application in the human body. Several biocompatible platforms such as silicon/gold/ platinum/titanium components and biodegradable polymers (for example, PLGA, PLA, polycaprolactone (PCL)) have been proven to be safe for in vivo applications. However, there is a limited number of functionalities that they can offer. It is still a grand challenge to make remotely triggerable DDSs that are fully biocompatible and biodegradable.

Aside from biocompatibility with the body environment, the DDS should also be compatible with the drugs. Otherwise, unwanted reactions might occur that damage the DDS and cause complications.

Effective cycle life. One of the most important parameters is the length of time that a wirelessly triggerable DDS can operate inside the body. This metric relies on three parameters: triggerability of the DDS as a function of time and number of successive excitations, biodegradability rate of the drug carrier and the amount of payload(s) released in every excitation.

The number of times a DDS can be triggered without losing performance should be much larger than the number of excitations it takes to fully deplete the DDS. In choosing the right stimulus for triggering DDSs, it is important to consider whether multiple DDSs are placed in close proximity. In this case, it is necessary to use stimuli with high spatial resolution and temporal control (for example, light).

Biodegradable DDSs release passively, setting the basal release rate, until the trigger increases the release rate. For certain applications, passive release can be harmful. A silicon chip DDS addresses this issue but is not biodegradable. On the contrary, the biodegradation rate of polymer-based platforms can be adjusted depending on the intended effective cycle life but at the cost of required higher excitation energy. In general, for biodegradable materials, sharp release transitions require high triggering energy, which can harm the healthy tissues.
The biodegradability rate of the drug carrier is most important when carrying potent payloads, or small payloads that need to travel long distances to reach the site of action. If the DDS degrades while still containing the payloads, it can cause severe adverse drug reactions. In the other extreme case, if after releasing all payloads on demand the DDS does not degrade (for example, the silicon microchip), it can cause long-term complications, especially when the device needs to be replaced frequently.

Off-target adverse effects. Every DDS has pros and cons, including the effects the drug and the stimulus can have on off-target tissues/ environments. For example, excitation of DDSs with magnetic/electromagnetic fields can be harmful to those who use a pacemaker. Potent chemotherapeutic agents can be detrimental to healthy tissues if the DDSs release inaccurate doses or release at unnecessary time intervals. Multistimuli platforms-DDSs that can be triggered with multiple endogenous and/or exogenous stimuli-can enhance the localization of the therapy. However, they are still at an early stage and more research is needed to validate their advantages over the already established single-stimulus techniques.

Some stimuli, such as ultraviolet and X-ray, are known to damage tissues and, for that reason, are often used in radiation therapy for cancer. It has been shown that synergistic effects can be obtained when ultraviolet- or X-ray-triggerable DDSs loaded with chemotherapeutic agents are used with radiation therapy (Fig. 6f). We believe there is plenty of room for using this strategy with other stimuli discussed in this Review.

Scalability for mass production. There is a rich literature on proof of concepts for various elegant DDSs that are fabricated from complex/long reaction routes and exotic precursors 7,70 . However, their scalability for mass production needs to be studied further.

\section{Outlook}

The field of drug delivery is interdisciplinary, incorporating polymer chemistry, medicine, materials science, biology and-increasingly-electrical engineering. As highlighted here, electrical engineering can play an influential role in the design of smart and functional DDSs, and considering recent advances in nanoelectronics, increased integration of electronics into smart DDSs can be expected in the coming years. Electronically powered, fully controllable DDSs could provide dynamic control over release profiles, as well as the timing of the release. Such systems could, for example, synchronize release-time intervals of chemotherapeutic regimens with the circadian rhythms of the patient. Adding sensing capabilities to the DDSs could also potentially enable fully automated DDSs that can sense the blood content, detect the change in the parameter of interest (sugar, for example), and trigger the system to release the appropriate payload. The functionality of DDSs in delivering therapeutic payloads to the target sites is of great importance in evaluating efficacy, but medication compliance and adherence are other factors that influence overall therapeutic efficacy. The development of low-cost and highly functional DDSs that can reside in the body for extended periods (including life-long) could enhance public health and help reduce healthcare costs in the long run. The use of digital and electronic systems is among the most promising approaches to achieving such goals.

Received: 14 October 2020; Accepted: 16 June 2021; Published online: 22 July 2021

\section{References}

1. Sennoga, C. A. et al. Microbubble-mediated ultrasound drug-delivery and therapeutic monitoring. Expert Opin. Drug Deliv. 14, 1031-1043 (2017).

2. Al Sawaftah, N. M. \& Husseini, G. A. Ultrasound-mediated drug delivery in cancer therapy: a review. J. Nanosci. Nanotechnol. 20, 7211-7230 (2020). 
3. Jain, A., Tiwari, A., Verma, A. \& Jain, S. K. Ultrasound-based triggered drug delivery to tumors. Drug Deliv. Transl. Res. 8, 150-164 (2018)

4. Seah, B. C.-Q. \& Teo, B. M. Recent advances in ultrasound-based transdermal drug delivery. Int. J. Nanomed. 13, 7749-7763 (2018),

5. George, P. M. et al. Electrically controlled drug delivery from biotin-doped conductive polypyrrole. Adv. Mater. 18, 577-581 (2006).

6. Svirskis, D., Travas-Sejdic, J., Rodgers, A. \& Garg, S. Electrochemically controlled drug delivery based on intrinsically conducting polymers. J. Control. Release 146, 6-15 (2010).

7. Norris, M. D., Seidel, K. \& Kirschning, A. Externally induced drug release systems with magnetic nanoparticle carriers: an emerging field in nanomedicine. Adv. Ther. 2, 1800092 (2019).

8. Mertz, D., Sandre, O. \& Bégin-Colin, S. Drug releasing nanoplatforms activated by alternating magnetic fields. Biochim. Biophys. Acta Gen. Subj. 1861, 1617-1641 (2017).

9. Khan, A. N., Ermakov, A., Sukhorukov, G. \& Hao, Y. Radio frequency controlled wireless drug delivery devices. Appl. Phys. Rev. 6, 041301 (2019).

10. Wang, X. et al. Near-infrared photoresponsive drug delivery nanosystems for cancer photo-chemotherapy. J. Nanobiotechnol. 18, 108 (2020).

11. Ai, X., Mu, J. \& Xing, B. Recent advances of light-mediated theranostics. Theranostics 6, 2439-2457 (2016)

12. Mirvakili, S. M. \& Hunter, I. W. Artificial muscles: mechanisms applications, and challenges. Adv. Mater. 30, 1704407 (2018).

13. Ge, J., Neofytou, E., Cahill, T. J., Beygui, R. E. \& Zare, R. N. Drug release from electric-field-responsive nanoparticles. ACS Nano 6 , 227-233 (2012).

14. Kagatani, S. et al. Electroresponsive pulsatile depot delivery of insulin from poly(dimethylaminopropylacrylamide) gel in rats. J. Pharm. Sci. 86 1273-1277 (1997).

15. Qu, J., Zhao, X., Ma, P. X. \& Guo, B. Injectable antibacterial conductive hydrogels with dual response to an electric field and $\mathrm{pH}$ for localized "smart" drug release. Acta Biomater. 72, 55-69 (2018)

16. Jeon, G., Yang, S. Y., Byun, J. \& Kim, J. K. Electrically actuatable smart nanoporous membrane for pulsatile drug release. Nano Lett. 11, 1284-1288 (2011).

17. Pillay, V. et al. A review of integrating electroactive polymers as responsive systems for specialized drug delivery applications. J. Biomed. Mater. Res. A 102, 2039-2054 (2014).

18. Denet, A.-R., Vanbever, R. \& Préat, V. Skin electroporation for transdermal and topical delivery. Adv. Drug Deliv. Rev. 56, 659-674 (2004).

19. Electroporation Protocols: Preclinical and Clinical Gene Medicine (Humana Press, 2014); https://doi.org/10.1007/978-1-4614-9632-8

20. Prausnitz, M. R. \& Langer, R. Transdermal drug delivery. Nat. Biotechnol. 26, 1261-1268 (2008).

21. Zhao, Y. L. et al. Induction of cytotoxic T-lymphocytes by electroporation-enhanced needle-free skin immunization. Vaccine $\mathbf{2 4}$ 1282-1290 (2006).

22. DNA medicines technology. INOVIO https://www.inovio.com/dnamedicines-technology/

23. Smith, T. R. F. et al. Immunogenicity of a DNA vaccine candidate for COVID-19. Nat. Commun. 11, 2601 (2020)

24. Pliquett, U. \& Weaver, J. C. Feasibility of an electrode-reservoir device for transdermal drug delivery by noninvasive skin electroporation. IEEE Trans. Biomed. Eng. 54, 536-538 (2007).

25. Ribas, J. et al. Arc stabilization in low-frequency square-wave electronic ballast for metal halide lamps. In 18th Annual IEEE Applied Power Electronics Conference and Exposition Vol. 2, 1179-1184 (IEEE, 2003).

26. Schlatter, S., Illenberger, P. \& Rosset, S. Peta-pico-Voltron: an open-source high voltage power supply. HardwareX 4, e00039 (2018).

27. Banga, A. K., Bose, S. \& Ghosh, T. K. Iontophoresis and electroporation: comparisons and contrasts. Int. J. Pharm. 179, 1-19 (1999).

28. Singhal, M. \& Kalia, Y. N. in Skin Permeation and Disposition of Therapeutic and Cosmeceutical Compounds (ed. Sugibayashi, K.) 165-182 (Springer, 2017); https://doi.org/10.1007/978-4-431-56526-0_14

29. Pikal, M. J. The role of electroosmotic flow in transdermal iontophoresis. Adv. Drug Deliv. Rev. 46, 281-305 (2001).

30. Subramony, J. A., Sharma, A. \& Phipps, J. B. Microprocessor controlled transdermal drug delivery. Int. J. Pharm. 317, 1-6 (2006).

31. Morrel, E. M., Spruance, S. L., Goldberg, D. I. \& Iontophoretic Acyclovir Cold Sore Study Group. Topical iontophoretic administration of acyclovir for the episodic treatment of herpes labialis: a randomized, double-blind, placebo-controlled, clinic-initiated trial. Clin. Infect. Dis. 43, 460-467 (2006)..

32. Wu, C. et al. Self-powered iontophoretic transdermal drug delivery system driven and regulated by biomechanical motions. Adv. Funct. Mater. 30 , 1907378 (2020).

33. Chaturvedula, A. et al. Dermal, subdermal, and systemic concentrations of granisetron by iontophoretic delivery. Pharm. Res. 22, 1313-1319 (2005).
34. Zempsky, W. T., Sullivan, J., Paulson, D. M. \& Hoath, S. B. Evaluation of a low-dose lidocaine iontophoresis system for topical anesthesia in adults and children: a randomized, controlled trial. Clin. Ther. 26, 1110-1119 (2004)

35. Kreyden, O. P. Iontophoresis for palmoplantar hyperhidrosis. J. Cosmet. Dermatol. 3, 211-214 (2004)

36. Beauchamp, M. \& Lands, L. C. Sweat-testing: a review of current technical requirements. Pediatr. Pulmonol. 39, 507-511 (2005).

37. Mayes, S. \& Ferrone, M. Fentanyl $\mathrm{HCl}$ patient-controlled iontophoretic transdermal system for the management of acute postoperative pain. Ann. Pharmacother. 40, 2178-2186 (2006).

38. Tamada, J. A. et al. Noninvasive glucose monitoring: comprehensive clinica results. JAMA 282, 1839-1844 (1999).

39. Perez, V. L., Wirostko, B., Korenfeld, M., From, S. \& Raizman, M. Ophthalmic drug delivery using iontophoresis: recent clinical applications. J. Ocul. Pharmacol. Ther. 36, 75-87 (2019).

40. Byrne, J. D. et al. Local iontophoretic administration of cytotoxic therapies to solid tumors. Sci. Transl. Med. 7, 273 ral4 (2015).

41. Byrne, J. D., Yeh, J. J. \& DeSimone, J. M. Use of iontophoresis for the treatment of cancer. J. Control. Release 284, 144-151 (2018).

42. Mitragotri, S. Synergistic effect of enhancers for transdermal drug delivery Pharm. Res. 17, 1354-1359 (2000).

43. Kirson, E. D. et al. Alternating electric fields arrest cell proliferation in animal tumor models and human brain tumors. Proc. Natl Acad. Sci. USA 104, 10152-10157 (2007).

44. Benson, L. Tumor treating fields technology: alternating electric field therapy for the treatment of solid tumors. Semin. Oncol. Nurs. 34, 137-150 (2018).

45. Kirson, E. D. et al. Chemotherapeutic treatment efficacy and sensitivity are increased by adjuvant alternating electric fields (TTFields). BMC Med. Phys. 9, 1 (2009).

46. Kost, J., Wolfrum, J. \& Langer, R. Magnetically enhanced insulin release in diabetic rats. J. Biomed. Mater. Res. 21, 1367-1373 (1987).

47. Zhao, X. et al. Active scaffolds for on-demand drug and cell delivery. Proc. Natl Acad. Sci. USA 108, 67-72 (2011).

48. Qin, J. et al. Injectable superparamagnetic ferrogels for controlled release of hydrophobic drugs. Adv. Mater. 21, 1354-1357 (2009).

49. Peiris, P. M. et al. Enhanced delivery of chemotherapy to tumors using a multicomponent nanochain with radio-frequency-tunable drug release. ACS Nano 6, 4157-4168 (2012).

50. Murthy, S. N., Sammeta, S. M. \& Bowers, C. Magnetophoresis for enhancing transdermal drug delivery: mechanistic studies and patch design. J. Control. Release 148, 197-203 (2010).

51. Qiu, Y. et al. Magnetic forces enable controlled drug delivery by disrupting endothelial cell-cell junctions. Nat. Commun. 8, 15594 (2017).

52. Chen, X.-Z. et al. Recent developments in magnetically driven micro- and nanorobots. Appl. Mater. Today 9, 37-48 (2017).

53. Kummer, M. P. et al. OctoMag: an electromagnetic system for 5-DOF wireless micromanipulation. IEEE Trans. Robot. 26, 1006-1017 (2010).

54. Li, J., Esteban-Fernández de Ávila, B., Gao, W., Zhang, L. \& Wang, J. Micro/ nanorobots for biomedicine: delivery, surgery, sensing, and detoxification. Sci. Robot. 2, eaam6431 (2017).

55. Nelson, B. J., Kaliakatsos, I. K. \& Abbott, J. J. Microrobots for minimally invasive medicine. Annu. Rev. Biomed. Eng. 12, 55-85 (2010)

56. Qiu, F. et al. Magnetic helical microswimmers functionalized with lipoplexes for targeted gene delivery. Adv. Funct. Mater. 25, 1666-1671 (2015)

57. Servant, A., Qiu, F., Mazza, M., Kostarelos, K. \& Nelson, B. J. Controlled in vivo swimming of a swarm of bacteria-like microrobotic flagella. Adv. Mater. 27, 2981-2988 (2015).

58. Felfoul, O. et al. Magneto-aerotactic bacteria deliver drug-containing nanoliposomes to tumour hypoxic regions. Nat. Nanotechnol. 11, 941-947 (2016).

59. Erkoc, P. et al. Mobile microrobots for active therapeutic delivery. Adv. Ther 2, 1800064 (2019)

60. Mirvakili, S. M., Sim, D., Hunter, I. W. \& Langer, R. Actuation of untethered pneumatic artificial muscles and soft robots using magnetically induced liquid-to-gas phase transitions. Sci. Robot. 5, eaaz4239 (2020).

61. Golovin, Y. I. et al. Towards nanomedicines of the future: remote magneto-mechanical actuation of nanomedicines by alternating magnetic fields. J. Control. Release 219, 43-60 (2015).

62. Das, P., Colombo, M. \& Prosperi, D. Recent advances in magnetic fluid hyperthermia for cancer therapy. Colloids Surf. B 174, 42-55 (2019).

63. Schuerle, S., Dudani, J. S., Christiansen, M. G., Anikeeva, P. \& Bhatia, S. N. Magnetically actuated protease sensors for in vivo tumor profiling. Nano Lett. 16, 6303-6310 (2016)

64. Shirmardi Shaghasemi, B., Virk, M. M. \& Reimhult, E. Optimization of magneto-thermally controlled release kinetics by tuning of magnetoliposome composition and structure. Sci. Rep. 7, 7474 (2017) 
65. $\mathrm{Bi}, \mathrm{H}$. et al. Current developments in drug delivery with thermosensitive liposomes. Asian J. Pharm. Sci. 14, 365-379 (2019).

66. Mirvakili, S. M., Ngo, Q. P. \& Langer, R. Polymer nanocomposite microactuators for on-demand chemical release via high-frequency magnetic field excitation. Nano Lett. 20, 4816-4822 (2020).

67. Lee, J. et al. Flexible, sticky, and biodegradable wireless device for drug delivery to brain tumors. Nat. Commun. 10, 5205 (2019).

68. Kim, Y.-J., Ebara, M. \& Aoyagi, T. A smart hyperthermia nanofiber with switchable drug release for inducing cancer apoptosis. Adv. Funct. Mater. 23, 5753-5761 (2013).

69. Tang, L. et al. Poly( $N$-isopropylacrylamide)-based smart hydrogels: design, properties and applications. Prog. Mater. Sci. 115, 100702 (2021).

70. Wang, Y. \& Kohane, D. S. External triggering and triggered targeting strategies for drug delivery. Nat. Rev. Mater. 2, 17020 (2017).

71. Garcia-Pinel, B. et al. Magnetically active pNIPAM nanosystems as temperature-sensitive biocompatible structures for controlled drug delivery. Artif. Cells Nanomed. Biotechnol. 48, 1022-1035 (2020).

72. Walker, M. et al. Magnetically triggered release of entrapped bioactive proteins from thermally responsive polymer-coated iron oxide nanoparticles for stem-cell proliferation. ACS Appl. Nano Mater. 3, 5008-5013 (2020).

73. Hoare, T. et al. A magnetically triggered composite membrane for on-demand drug delivery. Nano Lett. 9, 3651-3657 (2009).

74. Hoare, T. et al. Magnetically triggered nanocomposite membranes: a versatile platform for triggered drug release. Nano Lett. 11, 1395-1400 (2011).

75. Zaloga, J. et al. Development of a lauric acid/albumin hybrid iron oxide nanoparticle system with improved biocompatibility. Int. J. Nanomed. 9, 4847-4866 (2014)

76. Mahmoudi, K., Bouras, A., Bozec, D., Ivkov, R. \& Hadjipanayis, C. Magnetic hyperthermia therapy for the treatment of glioblastoma: a review of the therapy's history, efficacy and application in humans. Int. J. Hyperth. 34, 1316-1328 (2018).

77. Mirvakili, S. M., Broderick, K. \& Langer, R. S. A new approach for microfabrication of printed circuit boards with ultrafine traces. ACS Appl. Mater. Interfaces 11, 35376-35381 (2019).

78. Chopra, R. et al. Employing high-frequency alternating magnetic fields for the non-invasive treatment of prosthetic joint infections. Sci. Rep. 7, 7520 (2017)

79. Mohapatra, J., Xing, M. \& Liu, J. P. Inductive thermal effect of ferrite magnetic nanoparticles. Materials 12, 3208 (2019).

80. Monge, M., Lee-Gosselin, A., Shapiro, M. G. \& Emami, A. Localization of microscale devices in vivo using addressable transmitters operated as magnetic spins. Nat. Biomed. Eng. 1, 736-744 (2017).

81. Edelman, E. R., Kost, J., Bobeck, H. \& Langer, R. Regulation of drug release from polymer matrices by oscillating magnetic fields. J. Biomed. Mater. Res. 19, 67-83 (1985).

82. Medical Device Radiocommunications Service (MedRadio) Federal Communications Commission https://www.fcc.gov/medical-deviceradiocommunications-service-medradio (2011).

83. Santini, J. T., Cima, M. J. \& Langer, R. A controlled-release microchip. Nature 397, 335-338 (1999).

84. Farra, R. et al. First-in-human testing of a wirelessly controlled drug delivery microchip. Sci. Transl. Med. 4, 122ra21 (2012).

85. Yang, Y.-J. et al. A release-on-demand wireless CMOS drug delivery SoC based on electrothermal activation technique. In 2009 IEEE International Solid-State Circuits Conference-Digest of Technical Papers 288-289,289a (IEEE, 2009); https://doi.org/10.1109/ISSCC.2009.4977421

86. Li, Y. et al. In vivo release from a drug delivery MEMS device. J. Control. Release 100, 211-219 (2004).

87. Lee, C. H. et al. Biological lipid membranes for on-demand, wireless drug delivery from thin, bioresorbable electronic implants. NPG Asia Mater. 7, e227 (2015).

88. Grayson, A. C. R. et al. Multi-pulse drug delivery from a resorbable polymeric microchip device. Nat. Mater. 2, 767-772 (2003).

89. Langer, R. \& Tirrell, D. A. Designing materials for biology and medicine. Nature 428, 487-492 (2004).

90. Smith, S. et al. Development of a miniaturised drug delivery system with wireless power transfer and communication. IET Nanobiotechnol. 1, 80-86 (2007)

91. Tang, T. B. et al. Implementation of wireless power transfer and communications for an implantable ocular drug delivery system. IET Nanobiotechnol. 2, 72-79 (2008).

92. Assawaworrarit, S., Yu, X. \& Fan, S. Robust wireless power transfer using a nonlinear parity-time-symmetric circuit. Nature 546, 387-390 (2017).

93. Assawaworrarit, S. \& Fan, S. Robust and efficient wireless power transfer using a switch-mode implementation of a nonlinear parity-time symmetric circuit. Nat. Electron. 3, 273-279 (2020).

94. Nadeau, P. et al. Prolonged energy harvesting for ingestible devices. Nat. Biomed. Eng. 1, 0022 (2017).
95. Mirvakili, S. M. \& Hunter, I. W. Vertically aligned niobium nanowire arrays for fast-charging micro-supercapacitors. Adv. Mater. 29, 1700671 (2017).

96. Bandodkar, A. J. et al. Sweat-activated biocompatible batteries for epidermal electronic and microfluidic systems. Nat. Electron. 3, 554-562 (2020).

97. Talla, V. et al. Powering the next billion devices with wi-fi. In Proc. 11th ACM Conference on Emerging Networking Experiments and Technologies 4 (ACM, 2015); https://doi.org/10.1145/2716281.2836089

98. Hashemi, M. R. M. et al. A flexible phased array system with low areal mass density. Nat. Electron. 2, 195-205 (2019).

99. Lo, R. et al. A passive MEMS drug delivery pump for treatment of ocular diseases. Biomed. Microdevices 11, 959-970 (2009).

100. Samad, M. F. \& Kouzani, A. Z. Design and analysis of a low actuation voltage electrowetting-on-dielectric microvalve for drug delivery applications. In 2014 36th Annual International Conference of the IEEE Engineering in Medicine and Biology Society 4423-4426 (IEEE, 2014); https://doi.org/10.1109/EMBC.2014.6944605

101. Fong, J., Xiao, Z. \& Takahata, K. Wireless implantable chip with integrated nitinol-based pump for radio-controlled local drug delivery. Lab Chip 15 1050-1058 (2015)

102. Nafea, M., Nawabjan, A. \& Mohamed Ali, M. S. A wirelessly-controlled piezoelectric microvalve for regulated drug delivery. Sens. Actuators A 279, 191-203 (2018).

103. Jónasson, S. P., Zhurbenko, V. \& Johansen, T. K. Microwave assisted drug delivery. In 2014 XXXIth URSI General Assembly and Scientific Symposium (URSI GASS) 1-4 (IEEE, 2014); https://doi.org/10.1109/ URSIGASS.2014.6929196

104. Cherukuri, P., Glazer, E. S. \& Curley, S. A. Targeted hyperthermia using metal nanoparticles. Adv. Drug Deliv. Rev. 62, 339-345 (2010).

105. Glazer, E. S. \& Curley, S. A. Non-invasive radiofrequency ablation of malignancies mediated by quantum dots, gold nanoparticles and carbon nanotubes. Ther. Deliv. 2, 1325-1330 (2011).

106. Raoof, $M$. et al. Gold nanoparticles and radiofrequency in experimental models for hepatocellular carcinoma. Nanomed. Nanotechnol. Biol. Med. 10, 1121-1130 (2014)

107. Mironava, T., Arachchilage, V. T., Myers, K. J. \& Suchalkin, S. Gold nanoparticles and radio frequency field interactions: effects of nanoparticle size, charge, aggregation, radio frequency, and ionic background. Langmuir 33, 13114-13124 (2017)

108. Radio frequency (10 MHz-300 GHz). EMF-Portal https://www.emf-portal. org/en/cms/page/home/effects/radio-frequency

109. Light-emitting diodes (LEDs) in dermatology. SCMSJournal.com https:// www.scmsjournal.com/article/buy now/?id=259

110. Mathiyazhakan, M., Wiraja, C. \& Xu, C. A concise review of gold nanoparticles-based photo-responsive liposomes for controlled drug delivery. Nanomicro Lett. 10, 10 (2017).

111. Wu, L. et al. Remotely controlled drug release based on iron oxide nanoparticles for specific therapy of cancer. Colloids Surf. B 152, 440-448 (2017).

112. Al-Afyouni, M. H., Rohrabaugh, T. N., Al-Afyouni, K. F. \& Turro, C. $\mathrm{New} \mathrm{Ru}(\mathrm{II})$ photocages operative with near-IR light: new platform for drug delivery in the PDT window. Chem. Sci. 9, 6711-6720 (2018).

113. Timko, B. P. et al. Near-infrared-actuated devices for remotely controlled drug delivery. Proc. Natl Acad. Sci. USA 111, 1349-1354 (2014).

114. Zhan, C. et al. Phototriggered local anesthesia. Nano Lett. 16 177-181 (2016).

115. Yavuz, M. S. et al. Gold nanocages covered by smart polymers for controlled release with near-infrared light. Nat. Mater. 8, 935-939 (2009).

116. Barhoumi, A., Wang, W., Zurakowski, D., Langer, R. S. \& Kohane, D. S. Photothermally targeted thermosensitive polymer-masked nanoparticles. Nano Lett. 14, 3697-3701 (2014)

117. Mohammadi, M. R. et al. Nanomaterials engineering for drug delivery: a hybridization approach. J. Mater. Chem. B 5, 3995-4018 (2017).

118. Gao, S. et al. Biomimetic $\mathrm{O}_{2}$-evolving metal-organic framework nanoplatform for highly efficient photodynamic therapy against hypoxic tumor. Biomaterials 178, 83-94 (2018).

119. Luo, Z. et al. Self-monitoring artificial red cells with sufficient oxygen supply for enhanced photodynamic therapy. Sci. Rep. 6 , 23393 (2016).

120. Lin, C.-H., Aljuffali, I. A. \& Fang, J.-Y. Lasers as an approach for promoting drug delivery via skin. Expert Opin. Drug Deliv. 11, 599-614 (2014).

121. Younis, M. R. et al. Low power single laser activated synergistic cancer phototherapy using photosensitizer functionalized dual plasmonic photothermal nanoagents. ACS Nano 13, 2544-2557 (2019).

122. Li, J., Duan, H. \& Pu, K. Nanotransducers for near-infrared photoregulation in biomedicine. Adv. Mater. 31, 1901607 (2019).

123. Luo, Y.-L., Shiao, Y.-S. \& Huang, Y.-F. Release of photoactivatable drugs from plasmonic nanoparticles for targeted cancer therapy. ACS Nano 5 7796-7804 (2011). 
124. Xu, J., Zhou, X., Gao, Z., Song, Y.-Y. \& Schmuki, P. Visible-light-triggered drug release from $\mathrm{TiO}_{2}$ nanotube arrays: a controllable antibacterial platform. Angew. Chem. Int. Ed. 128, 603-607 (2016).

125. Shell, T. A. \& Lawrence, D. S. Vitamin B12: a tunable, long wavelength, light-responsive platform for launching therapeutic agents. Acc. Chem. Res. 48, 2866-2874 (2015).

126. Leonidova, A. et al. Photo-induced uncaging of a specific $\operatorname{Re}(\mathrm{I})$ organometallic complex in living cells. Chem. Sci. 5, 4044-4056 (2014).

127. Wang, Y. et al. Intravenous treatment of choroidal neovascularization by photo-targeted nanoparticles. Nat. Commun. 10, 804 (2019).

128. Linsley, C. S. \& Wu, B. M. Recent advances in light-responsive on-demand drug-delivery systems. Ther. Deliv. 8, 89-107 (2017).

129. Lendlein, A., Jiang, H., Jünger, O. \& Langer, R. Light-induced shape-memory polymers. Nature 434, 879-882 (2005).

130. Tong, R., Chiang, H. H. \& Kohane, D. S. Photoswitchable nanoparticles for in vivo cancer chemotherapy. Proc. Natl Acad. Sci. USA 110 , 19048-19053 (2013).

131. Hill, R. et al. Advances in kilovoltage X-ray beam dosimetry. Phys. Med. Biol. 59, R183-R231 (2014).

132. Deng, W. et al. Controlled gene and drug release from a liposomal delivery platform triggered by X-ray radiation. Nat. Commun. 9, 2713 (2018).

133. Sakar, F. et al. Nano drug delivery systems and gamma radiation sterilization. Pharm. Dev. Technol. 22, 775-784 (2017).
134. Tottori, S. et al. Magnetic helical micromachines: fabrication, controlled swimming, and cargo transport. Adv. Mater. 24, 811-816 (2012).

135. Amendola, V., Pilot, R., Frasconi, M., Maragò, O. M. \& Iatì, M. A. Surface plasmon resonance in gold nanoparticles: a review. J. Phys. Condens. Matter 29, 203002 (2017)

\section{Competing interests}

S.M.M. declares no competing interests. For a list of entities with which R.L. is involved, compensated or uncompensated, see: www.dropbox.com/s/yc3xqb5s8s94v7x/Rev\%20

Langer\%20COI.pdf?dl=0.

\section{Additional information}

Supplementary information The online version contains supplementary material available at https://doi.org/10.1038/s41928-021-00614-9.

Correspondence should be addressed to S.M.M. or R.L.

Peer review information Nature Electronics thanks Azita Emami and the other, anonymous, reviewer(s) for their contribution to the peer review of this work.

Reprints and permissions information is available at www.nature.com/reprints.

Publisher's note Springer Nature remains neutral with regard to jurisdictional claims in published maps and institutional affiliations.

(c) Springer Nature Limited 2021 